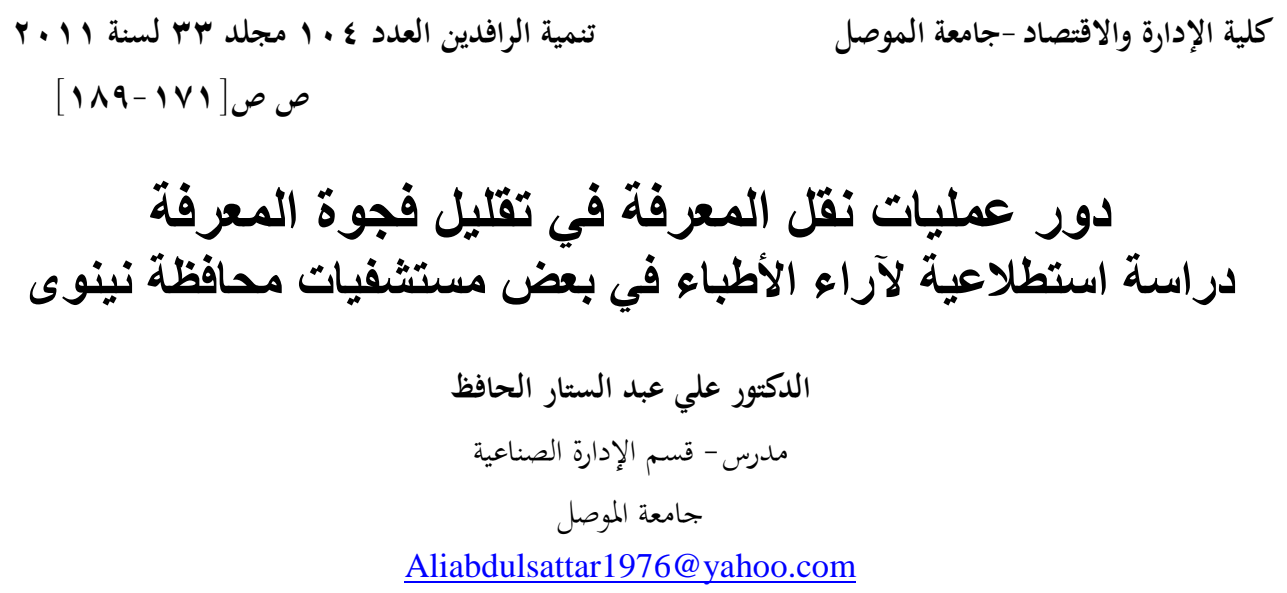

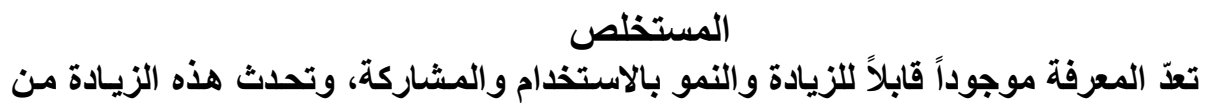

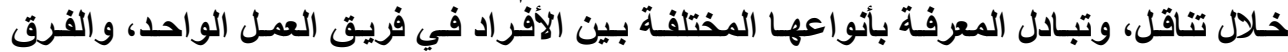

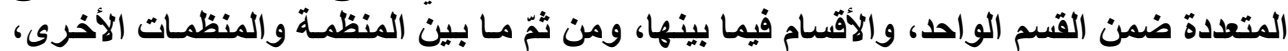

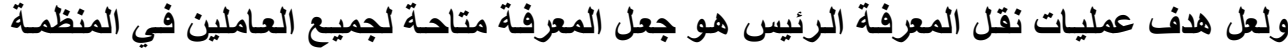

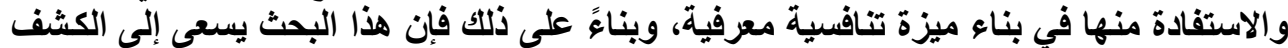

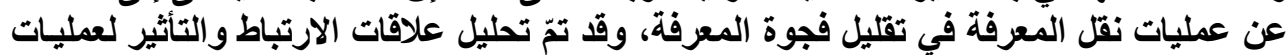

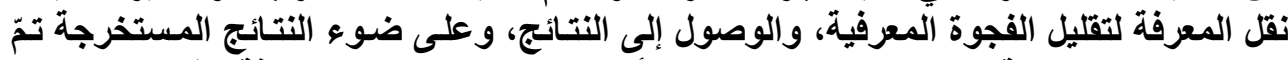

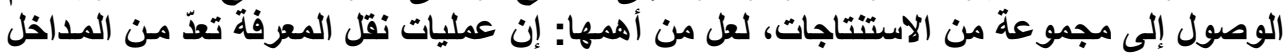

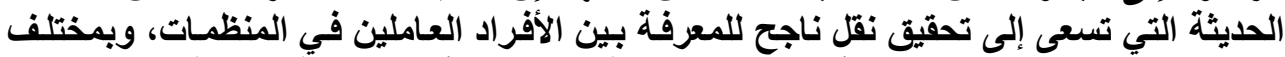

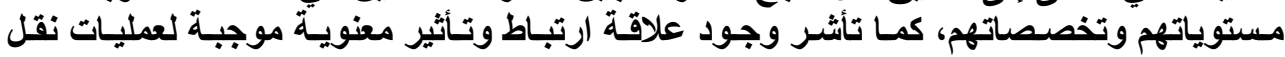

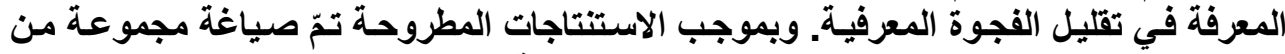
المقترحات التي يمكن تبنيها من قبل المستثفيات المبحوثة والتي والتي تهدف إلى تحسين عمليات نقل

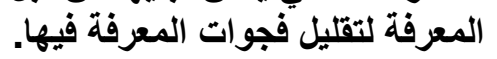

الكلمات المفتاحية: إدارة المعرفة، عمليات إدارة المعرفة، فجوة المعرفة، نقل المعرفة، عمليات

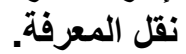

\title{
The Role of Knowledge Transfer Processes in Reducing Knowledge \\ Gap: A Pilot Study for Opinions of Doctors in Some of Nineveh Governorate Hospitals
}

\author{
Ali A. Al-Hafedh (PhD) \\ Lecturer \\ Department of Industrial Management \\ University of Mosul
}

\section{Abstract}

Knowledge is considered to be an existence able to increase and grow by using and participating in it. This increase happens through interact different types of knowledge 
among individuals within the same work group, among several groups within the same department, among departments themselves and then among organization with the others. Therefore; the principal aim of knowledge processes transferring is to make knowledge available for all workers in the organization and to benefit from it in building knowledgeable competitive feature. Thus, this research tries to unveil the processes of knowledge transferring in reducing the knowledge gap. It has analyzed the connection relations and its affect upon processes of knowledge transferring in reducing knowledge gap to reach the results. On the light of the findings, a group of conclusions has been reached; one of the important conclusions is that knowledge processes transferring have been considered one of the recent approaches which tries to obtain successful transferring of knowledge among individuals working in organizations. It also indicates that there is a connection relation and strong positive abstract affect on the processes of knowledge transferring in reducing knowledgeable gap. According to the above mentioned conclusions a group of suggestions which can be adopted by hospitals under research has been formulated. The aim is to improve the processes of knowledge transferring to reduce the knowledge gaps in it.

Key Words: Knowledge Management, Knowledge Management Processes, Knowledge Gap, Knowledge Transfer, Knowledge Transfer Processes.

المقدمة

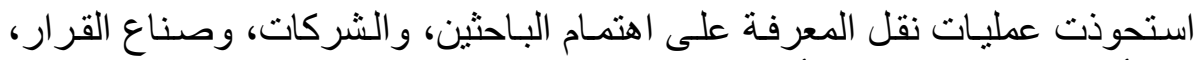

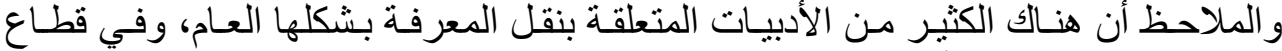
الرعاية الصحية هنالك تأكيد منز ايد على ضـرورة تسـيل نقل الممارسـات، ونتائج البحوث

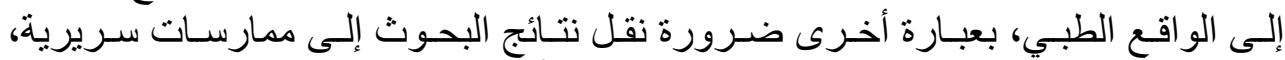

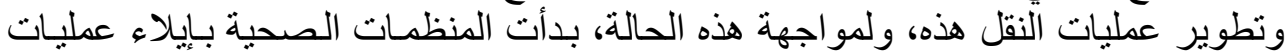
نقل المعرفة اهتمام وثركيز على وفق ما عرضته الدراسـات والبحوث في الواقع السريري

و الملاحظ أنه على الرغم من وجود إدارة معنية بالمعرفة (تعرف بـإدارة المعرفة) إلاّ

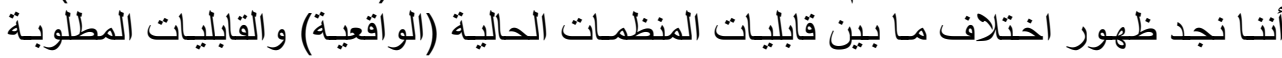
لتحقيق إدارة معرفة ناجحة في المنظمـات، و هذه الاختلافات والتباينات أطلات عليها فجوة المعرفة Knowledge Gap.

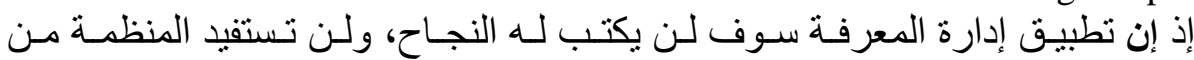

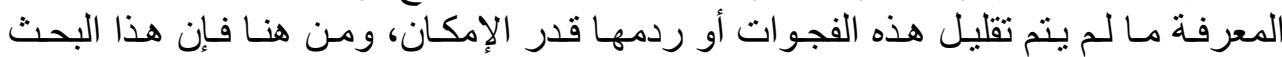
الأساس سبركز على دور عمليات نقل المعرفة في تقليل الفجوة المعرفية.

$$
\begin{aligned}
& \text { الإطار المنهجي } \\
& \text { مشكلة الاراسة الإنة } \\
& \text { تحتاج المنظمات الحديثة إلى المعرفة لإنتاج السلع وتقديم الخدمات، ولإيجـاد وتوزيع لئح } \\
& \text { المعرفة يتوجب أن يتم نقل هذه المعرفة بين العاملين بوصفهم منتجين للمعرفة المئ. }
\end{aligned}
$$

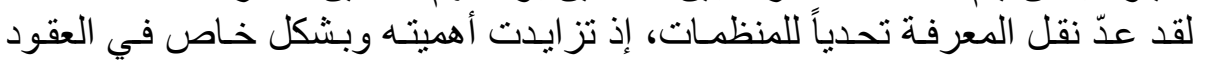

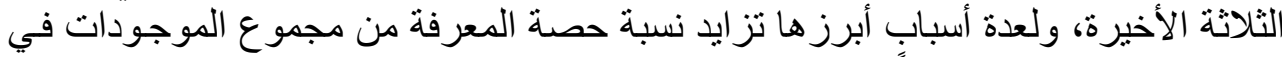

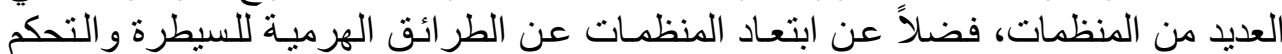




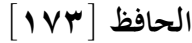

باتجاه الهياكل التنظيمية غير المركزية، وزيادة مشاركة العاملين في إطار النظم أو المداخل

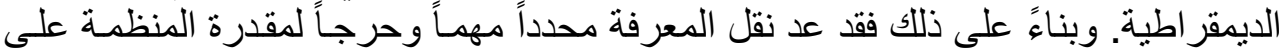

الحصول على ميزة تنافسية مستدامة.

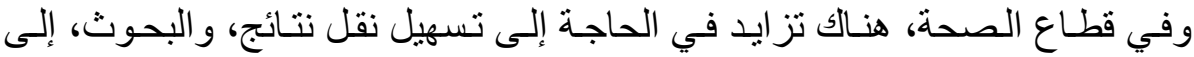

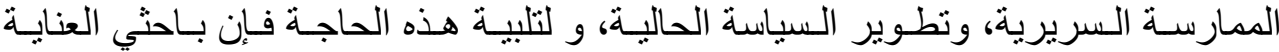

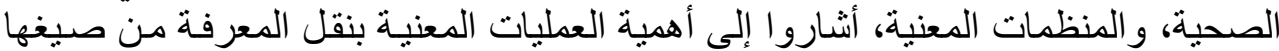

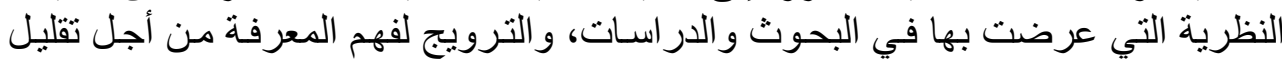
الفجوة المعرفية، وزيادة استفادة المرضى بما لألى الكادر الطبي من معرفة متر اكمة.

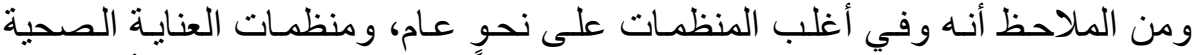

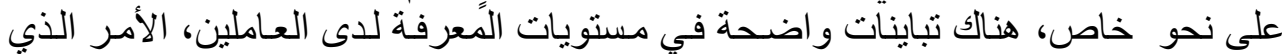

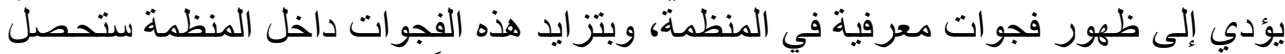

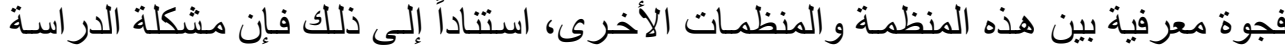
تبرز من خلال التساؤلات الآتية:

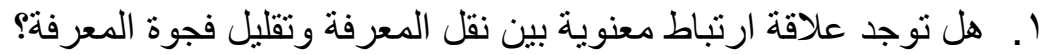

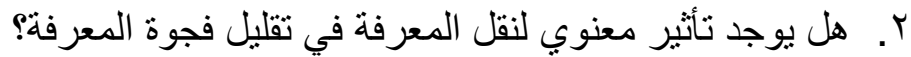

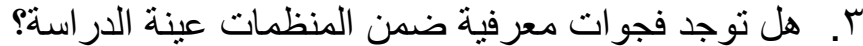
ع. هل يدرك المدر اء في المنظمات المبحوثة وجود فجوات معرفية في منظماتهج؟

أهمية الدراسة

تعد هذه الدر اسـة محاولـة لمعرفـة أثر نقل المعرفـة في الفجوة المعرفية، ومـا تؤديـه

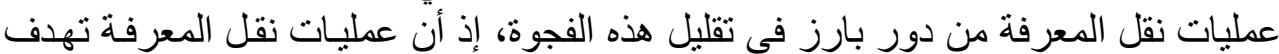

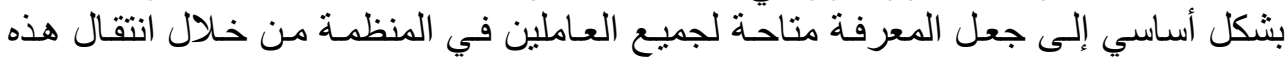

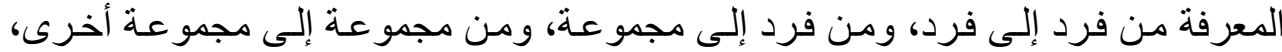

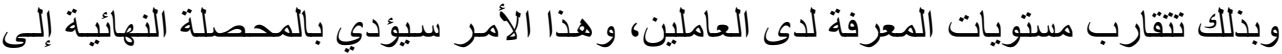

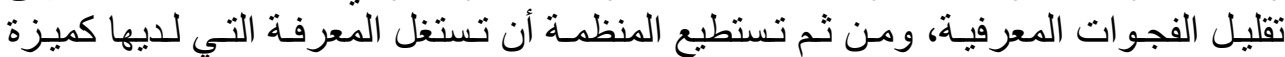

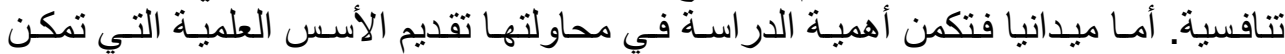

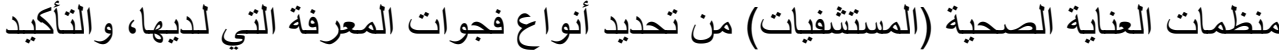

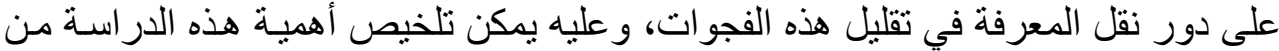

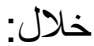

1. تحديد أثر نقل المعرفة في تقليل فجوة المعرفة في المنظمات المبحوثة.

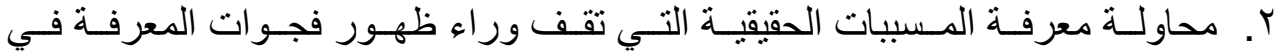

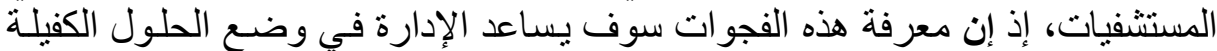

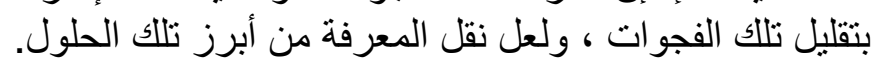

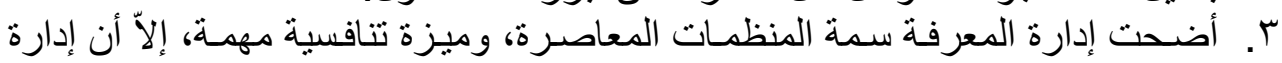

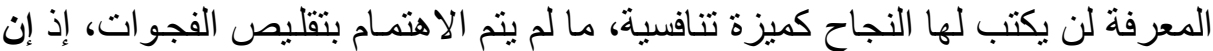
در اسة تقليص فجوات المعرفة لم يدرس بالثكل الذي يتلاءم وأهمية هذا الموضو الأهوع. 
يتمثل الهدف الرئيس للار اسة بتقييم الوضع الحالي الذي يشهده قطاع العناية الصحية،

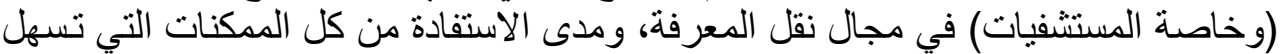

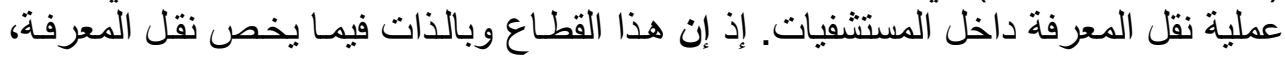

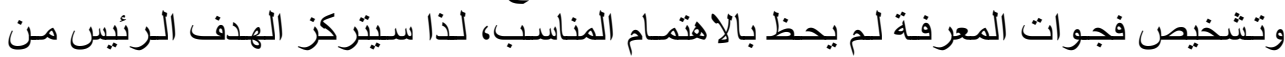

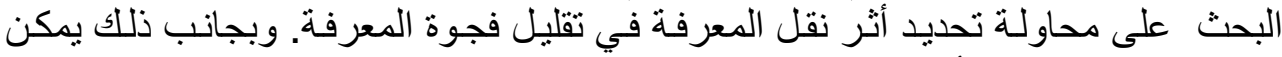
الإشارة إلى عدد من الأهداف التي ترمي الدار القة إلى الى تحقيقها:

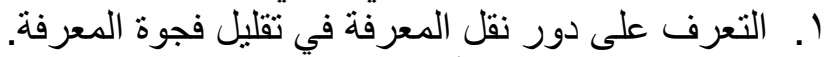

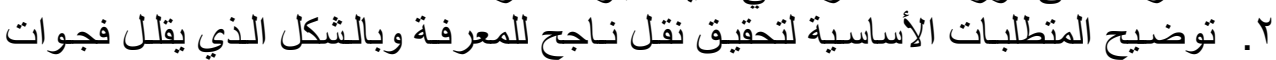
المعرفة، ويحد من تأثير اتها السلبية.

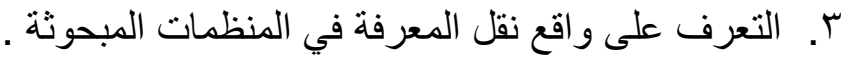
ع. تشخيص الفجوات المعرفية الموجودة في المستشفيات.

فرضيات الاراسة

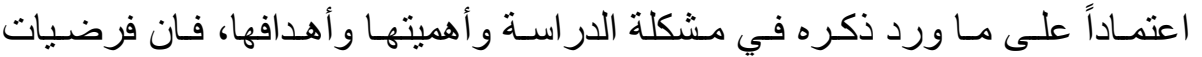

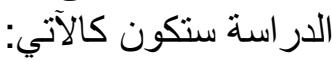
ا ـ. هنالك علاقة ارتباط معنوية بين نقل المعرفة وتقليل فجوة المعرفة. Y. . هنالك تأثثير معنوي لنقل المعرفة في تقليل فجوة المعرفة. منهج الدراسة

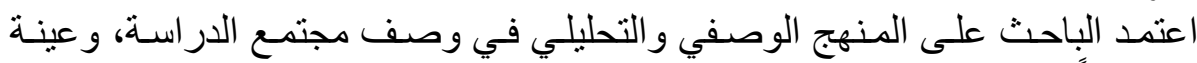
الدراسة، فضلاً عن تحليل العلاقة بين متغير العيل ات الدر استة. حدود الدراسة

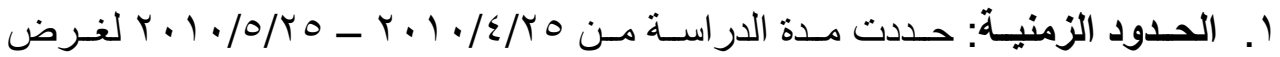
الحصول على البيانات اللازمة.

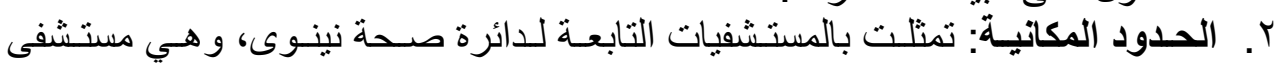

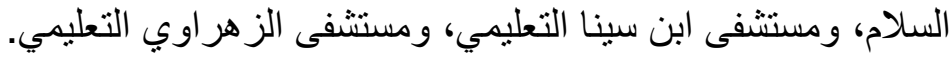

\section{أساليب جمع البيانات}

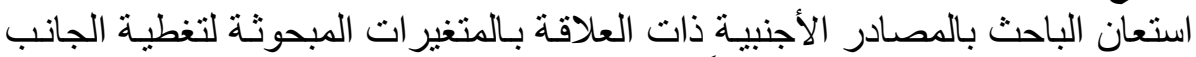

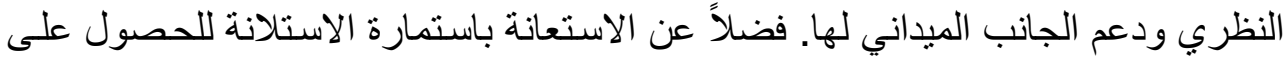

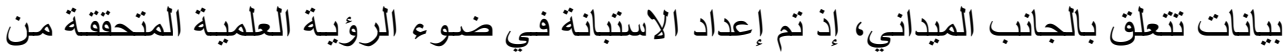

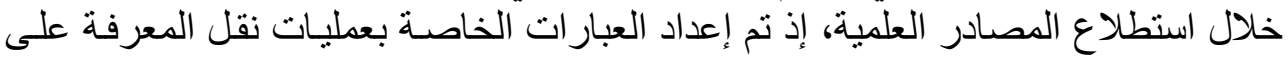

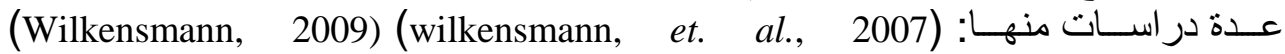
و (Ruse et. al., 2010) (Bratiainn et. al., 2009) وفي بّعد الفجوة المعرفية فقد تم إعداد

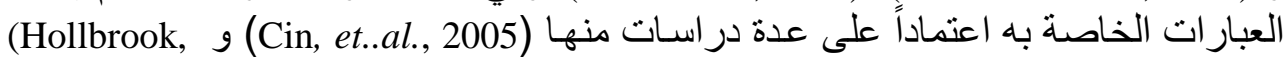




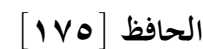

أ. معامل الارتباط البسيط والمتعدد لتحديد قوة علاقات الارتباط ومعنو ياتها بين عمليات نقل المعرفة وتقليل الفجوة المعرفية.

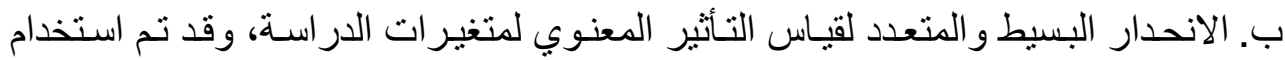

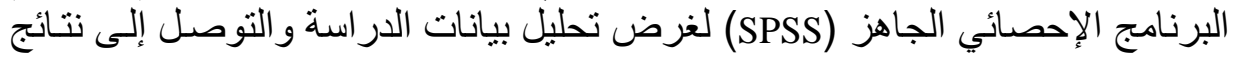

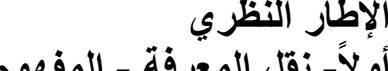

تحتاج المنظمات الحديثة إلى المعرفة لإنتاج السلع، وتقديم الخدمات، و لأجل تكوين

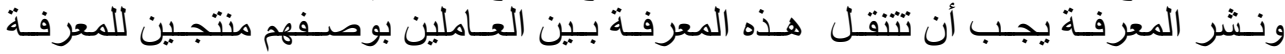

(Wilkesmann, et. al., 2009, 3) إن نقل المعرفة يصف كيف أن المعرفة والأفكار يتحركان من مصدر المعرفة إلى المستعملين المحتملين لتلك المعرفة.

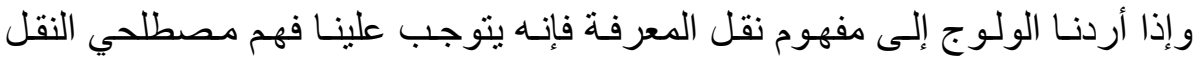
(Transfer)

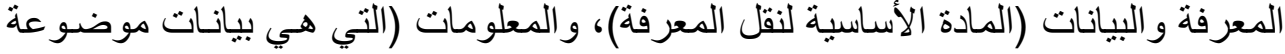

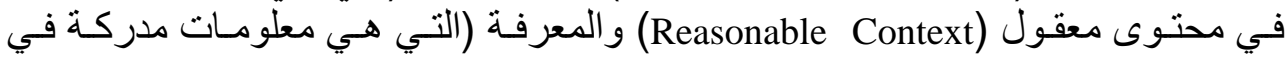

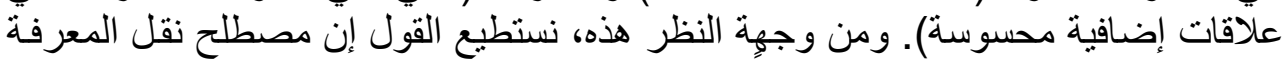

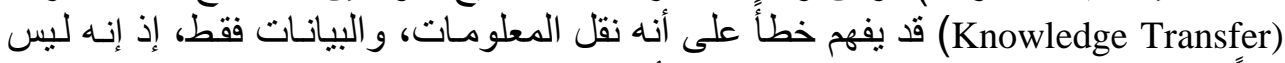

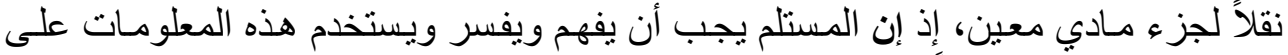

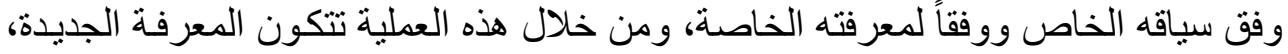
عبر تبادل المعرفة، و المعلومات، و البيانات (Wilkesmann, et. al., 2007, 5).

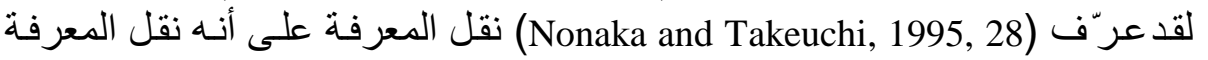
الضمنية (Tacit Knowledge)، و المعرفة الظاهرة (Explicit Knowledge) في حال التفاعل

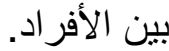

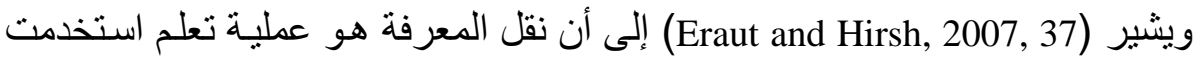

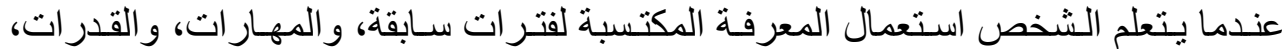

$$
\text { و الخبر ات، في حالات جديدة. }
$$

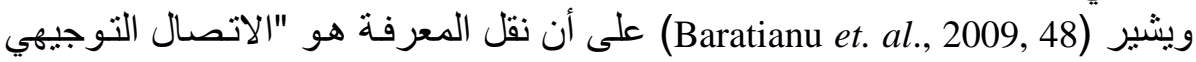
المركز للمعرفة بين العاملين، والمجمو عات، و المنظمـات التي يكون استخدام المعرفة فيها على شكل فهم إدر اكي يمكن من استقبال هذه المعرفة، ومن ثم استخدامها"، وكما في الشكل

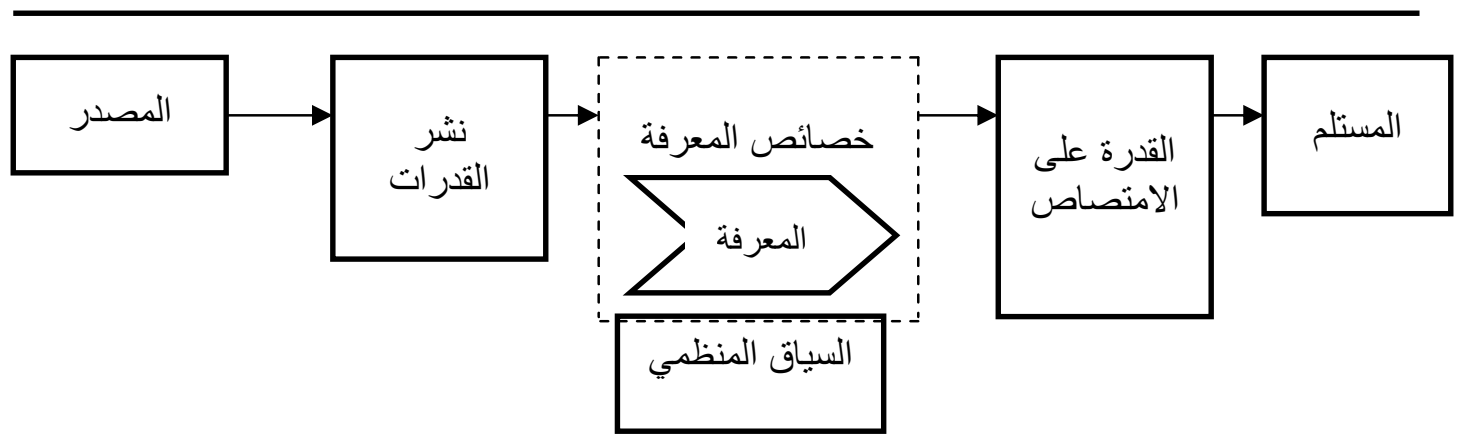




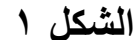 \\ مخطط لعملية نقل المعرفة}

Source: Bratinu, Constantin, Vas. Lache. Simon, Stancov, Vitalie, (2009), Knowledge Transfer Process in Romanian Multinational Companies, Review of International Comparative Management, Vol. (1) Issue (1).

إن نقل المعرفة هي العملية التي تتنقل فيها المعرفة من حامل المعرفة (Knowledge)

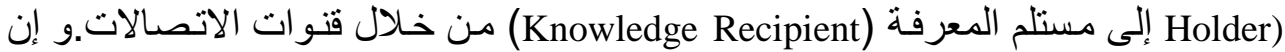

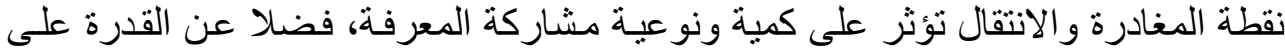

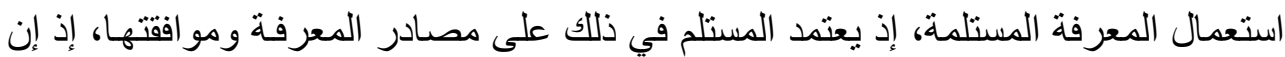

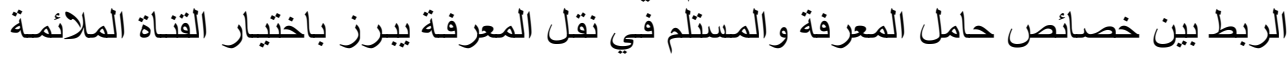
للنقل، إذ توجد قنوات متعددة للنقل ومنها (www.region s4research.eu; 2008, 4-5).

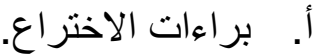
ب. ب. الاتصال بالجهات الأكاديمية.

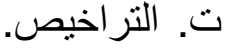
و الثكل r يوضح عملية نقل المعرفة.

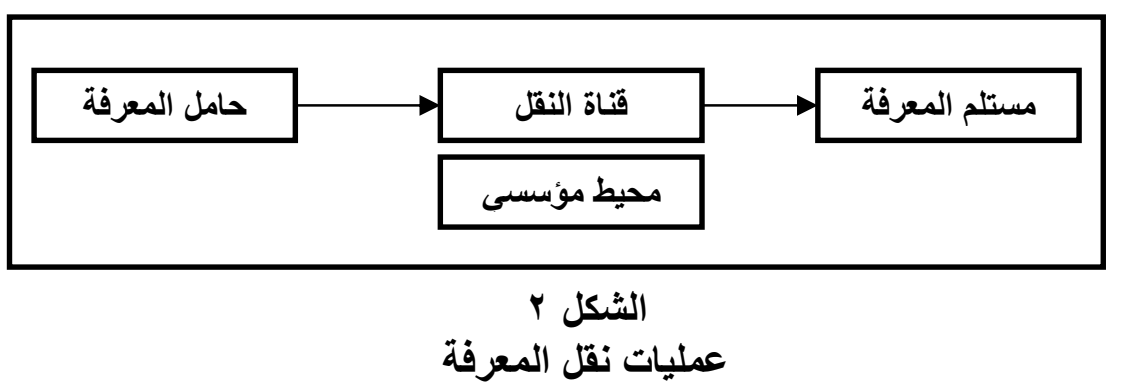

Source: www.region s4research- eu: 2008, 4-5.

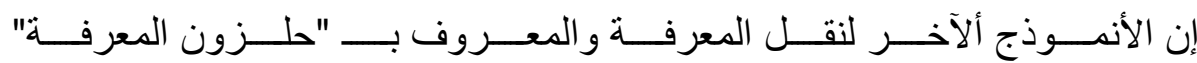
(Knowledge Spiral)

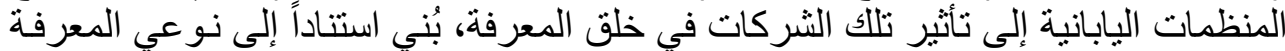

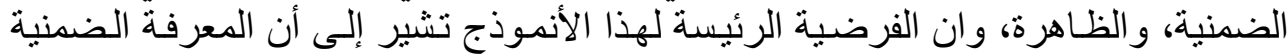

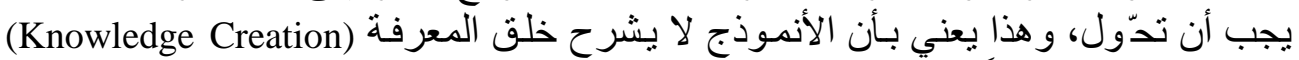

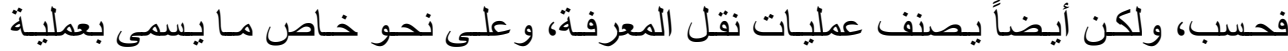
التحويل. لقد حدد (Nonaka and Takeuchi, 1995) أربع عمليات محددة لنقل المعرفة هي في

:(Elinfoo, 2005, 3-4)

ا ـ التجانس (socialization): (ضمنية إلى ضمنية) 


\section{[ivv] [ الحافظ}

وتحدث عندما يتبادل الأفر اد العاملون معرفة ضمنية تم تصنيفها أثتاء مرحلة النقل، التحا،

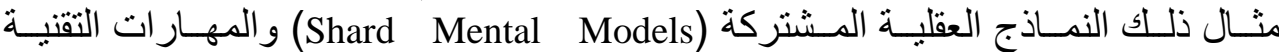
.(Technical Skills) r. التجسيد (الإبراز) (Externalization): (ضمنية إلى ظاهرة)

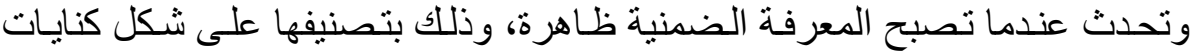

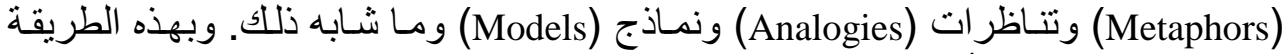

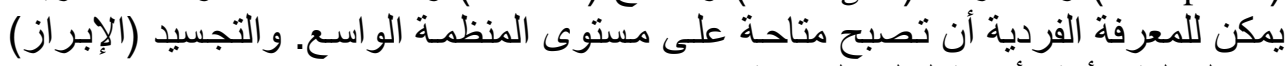
هي العملية الأكثر أهمية لخلق المعرفة المئ.

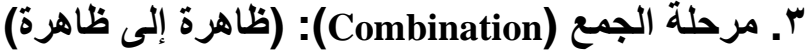

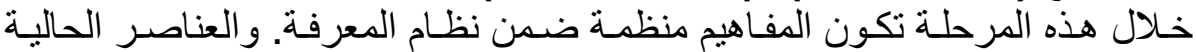

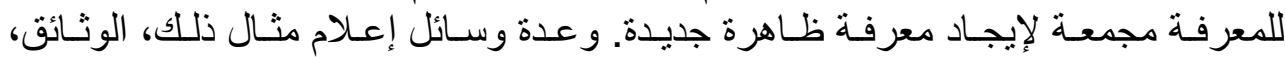
و الاجتماعات، و المكالمات الهاتفية، ودعم المزيج. ع. الامج أو التذويت (Internalization): (ظاهرة إلى ضمنية التية)

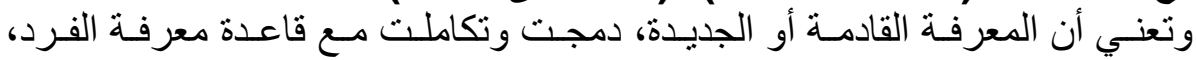
و الثكل ب يوضح العلاقة بين عمليات التحول.

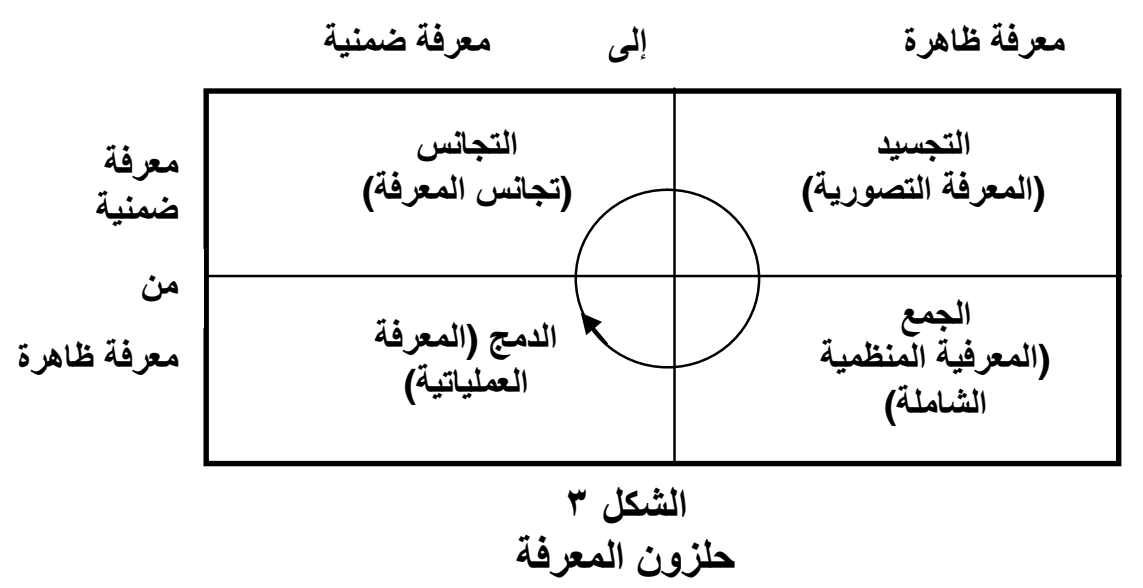

Source: Elinfoo, K. Harriet (2005), "Knowledge Creation \& Knowledge Transfer in Construction Organization in Tanzania, Doctoral Thesis, Royal in Statute of Technology Stockholm, Sweden. (www.kth.se. polopoly-f.com) 39-4 .

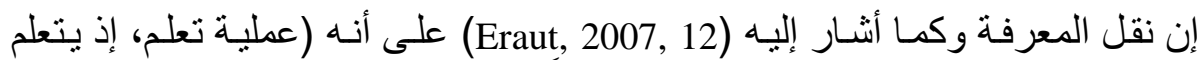

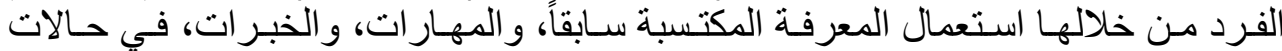

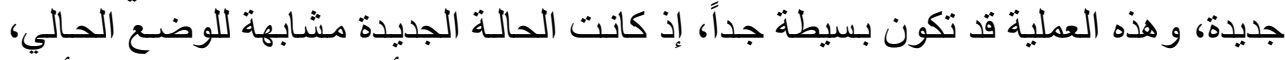

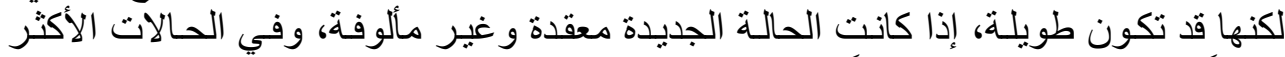
تعقيداً فإن عملية النقل أنموذجياً تتضمن خمس مر احل متر ابطة:

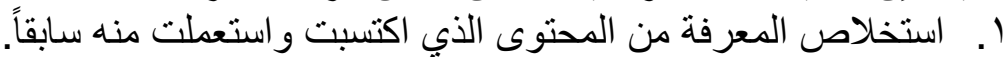

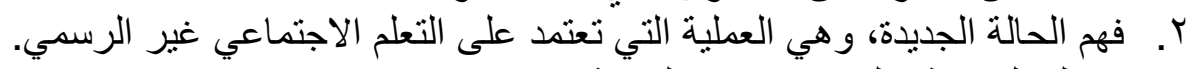

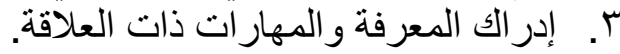


ع. ـ تحويل المعرفة و المهار ات ذات العلاقة إلى الحالة أو الوضع الجديد.

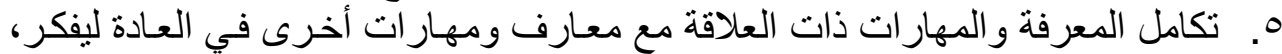
وينفذ، وينتقل إلى الحالة الجديدة.

إن نقل المعرفة وكما ير اه (Vote and Schiuma, 1999, 53-63) على إنى أنسه إستر اتيجية

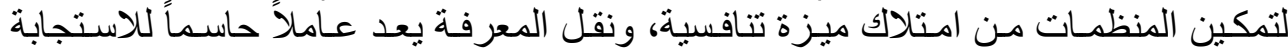
السريعة للتغيير الذي يواجه المنظمة، والإبداع، وتحقيق النجاح، و التفوق التنافسي.

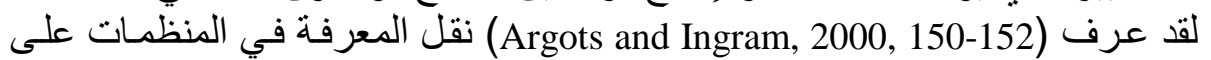

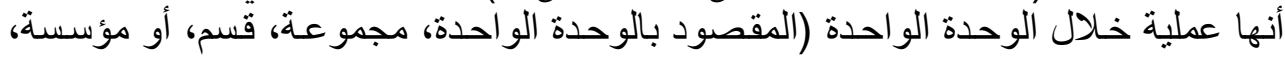

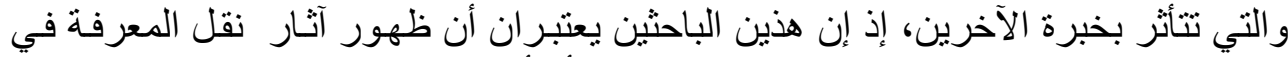

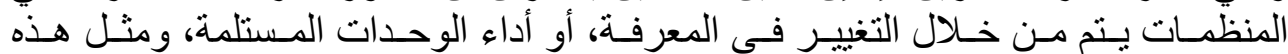

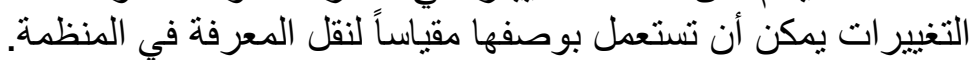
ويشير (Brochner and Waara, 2001, 422) إلى أن صفي فئة الانتقال (Transmission) صفة متأصلة وملازمة لنقل المعرفة، إذ أن فكرة نقل المعرفة قائمة على إرسال أحد الأفراد

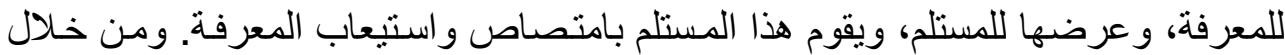

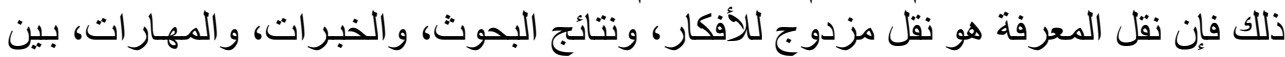

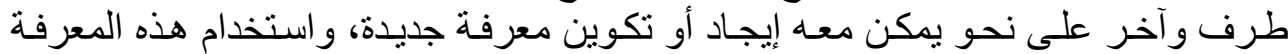

(Ruck, 2006, 35)

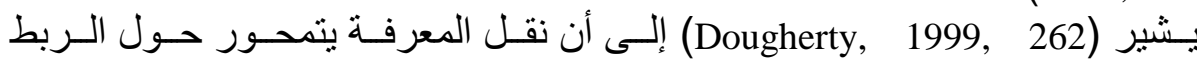
ولبيس حول التجميع (Connection)

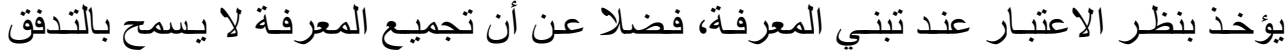

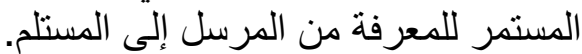

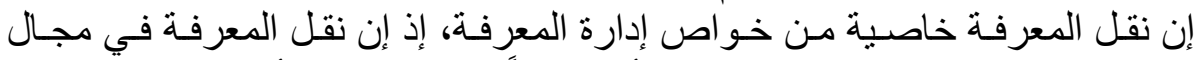

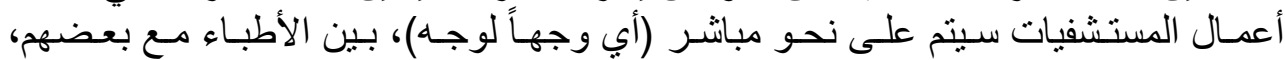

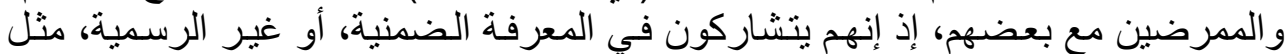

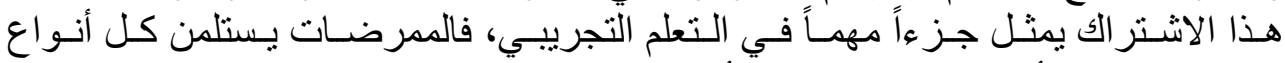

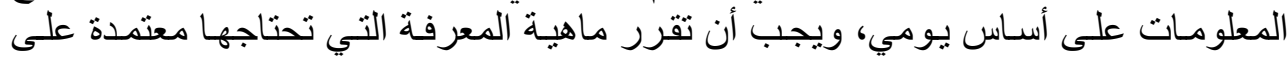

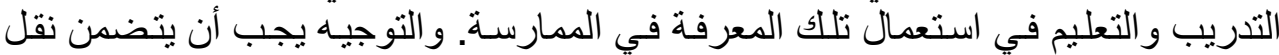

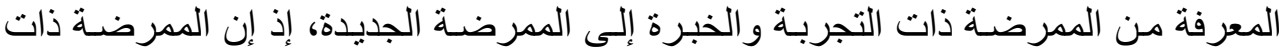

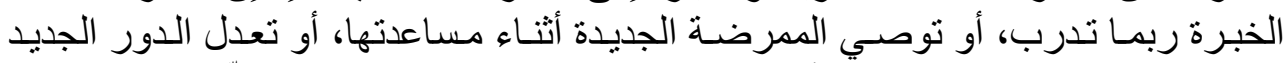

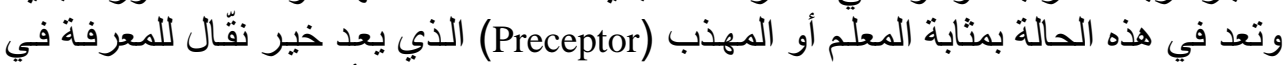

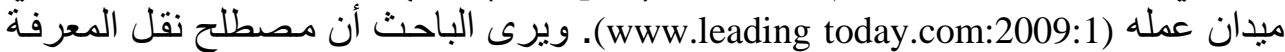

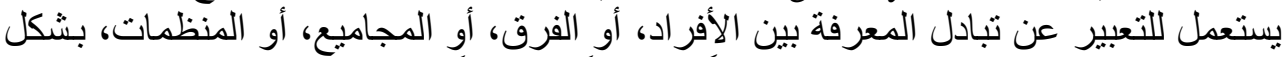

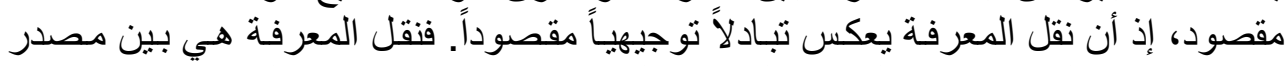
و اضح معرف للمعرفة ومستلم لهذه المعرفة، لها تركيز ، وهد هدف محدد على نحوِ واضح.

ثانياً - أهمية نقل المعرفة يعد نقل المعرفة تحدياً للمنظمـات، وتنتامت أهميته في العقود الثنلاثة الأخيرة، وذلك

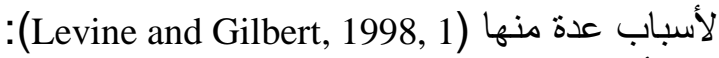
1. أصبحت المعرفة ذات نسبة متز ايدة في موجودات المنظمات. 


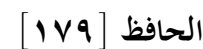

r. ابتعاد المنظمات عن الطر ائق الهرمية للسيطرة والرقابة باتجاه الهياكل غير الرسمية. r. ز زيادة تضمين (احتواء) العاملين.

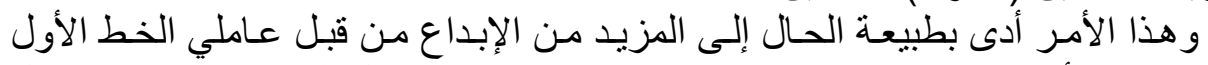

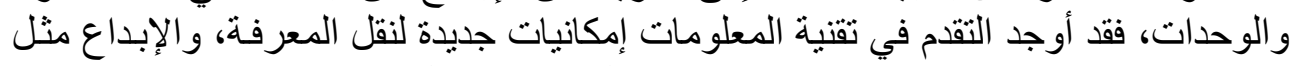

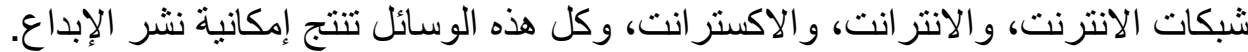

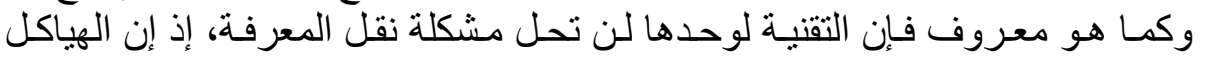

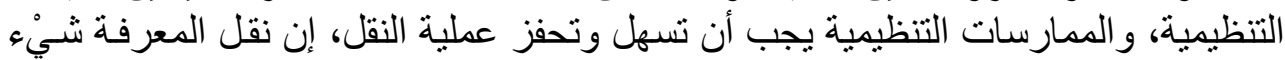

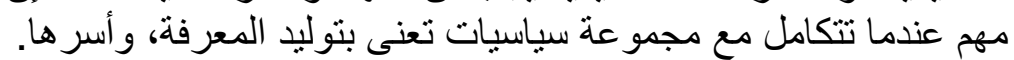

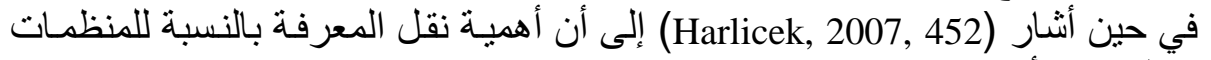

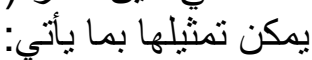

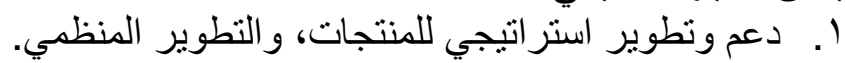

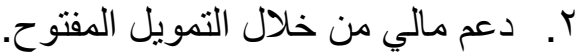

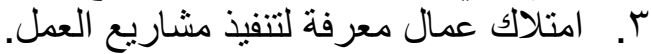

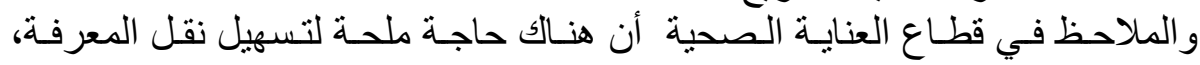

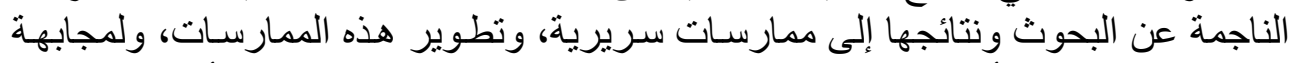

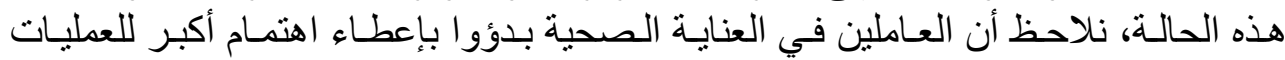

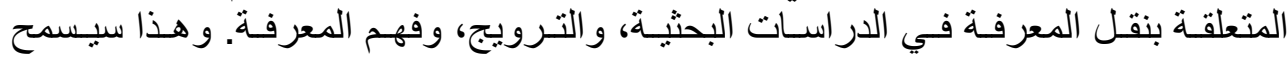

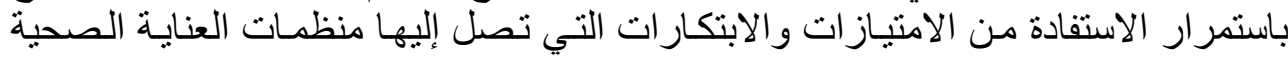
من ممارسات نقل المعرفة (http//:bluewirecst.tz0-com:2004,1).

ثنالثاً مراحل نقل المعرفة

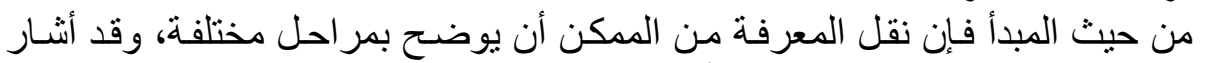
(Levin and Gilbert, 1998, 1-3)

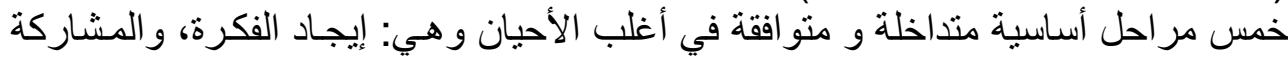
بالفكرة، وتقييم الفكرة، ونشر الفكرة، وتبني الفكرة.

ركزت العديد مـن الأدبيـات على الترويج للإبـداع، وتوجد جملـة مـن الاستفسارات

ا إيجاد الفكرة

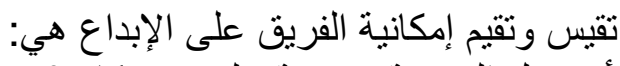

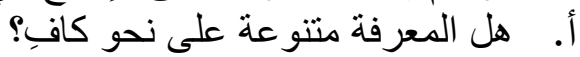
ب. هل موقف المجموعة عن المعرفة الخاصة بهم يتضمن الالتز ام بمـا يعرفونـه، و البحث عن مالا يعرفونه؟

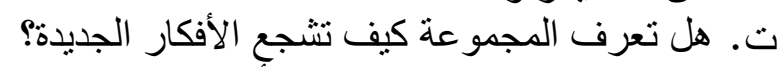

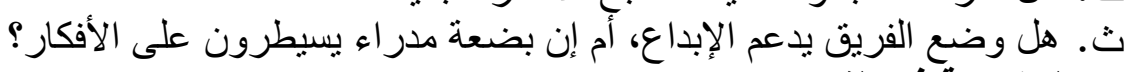

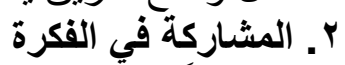

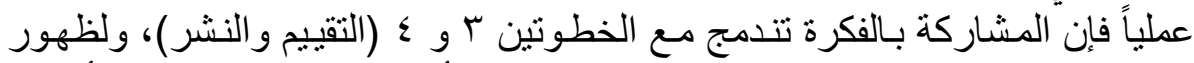

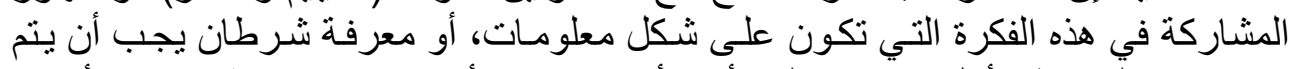

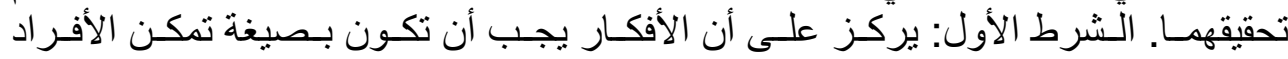

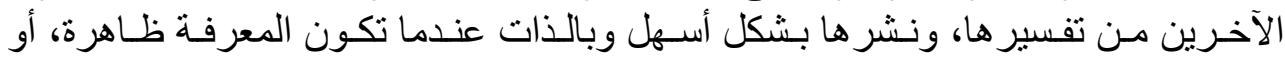


رسمية للعديد من المهار ات، والأفكار ، و هذا يتضمن تحويل الأفكار إلى صيغة مرمزة، مئهة

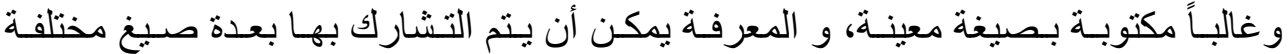

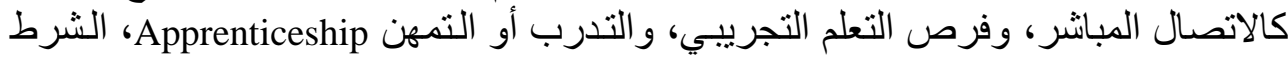

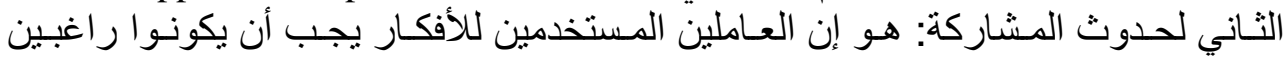

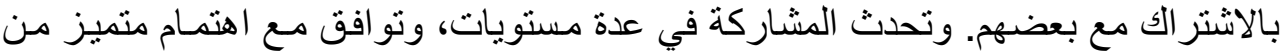

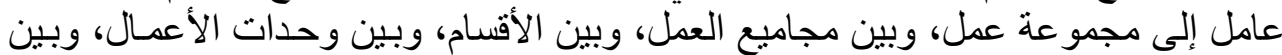

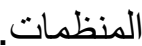

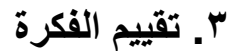

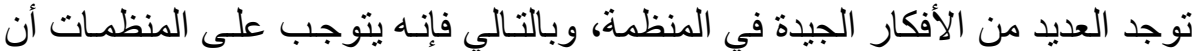

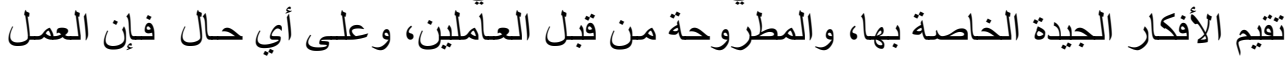

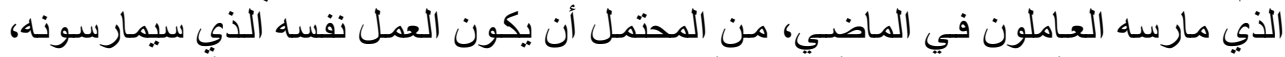

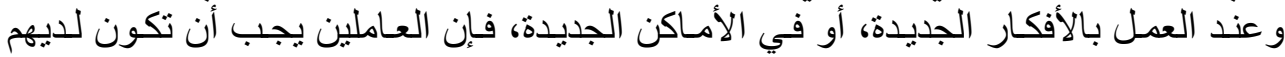

القابلية، و الحافز ، وتتو افر لديهم الهياكل للقيام بدر اسة شرعية الفكرة "Validation Study".

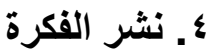

تثشير الأدبيات إلى أن المزيد من المعلومات، أفضل من المعلومات القليلة و المحدودة،

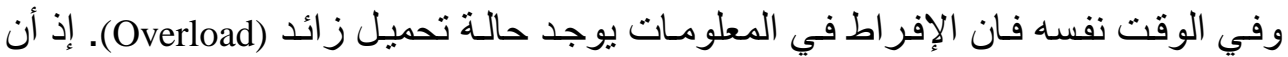

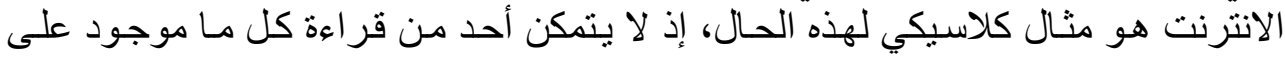

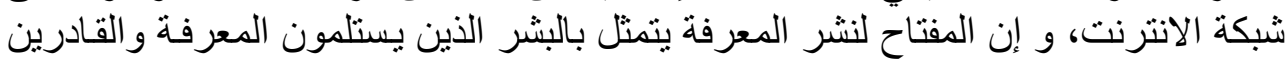

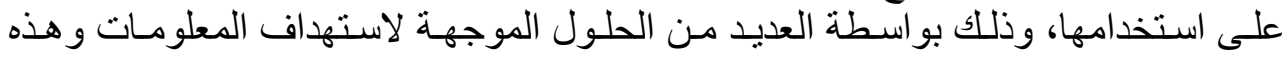

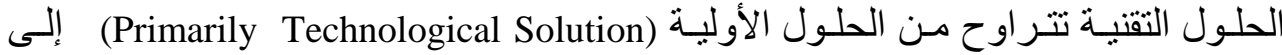

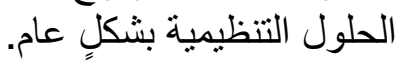

•. تبني الفكرة

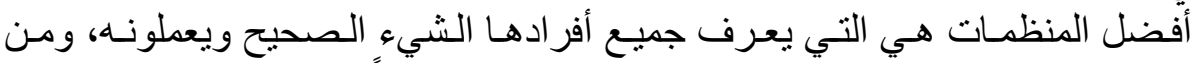

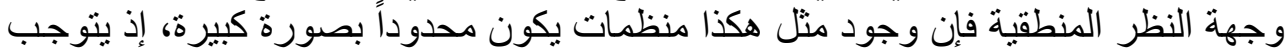

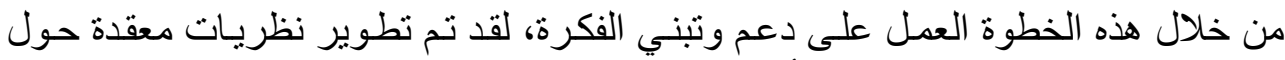

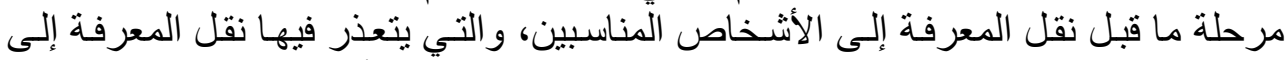

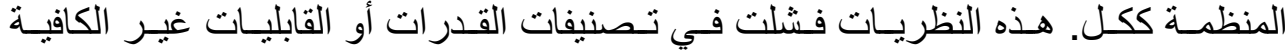

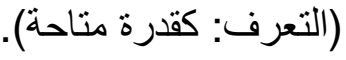

إن الظهور الأول لنظرية فجوة المعرفة كان من قبل (Tichenor, Donohue, oline)

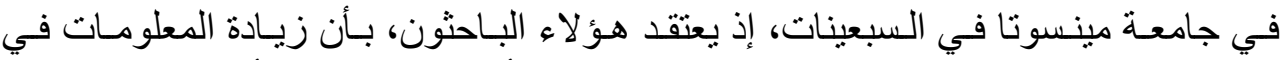

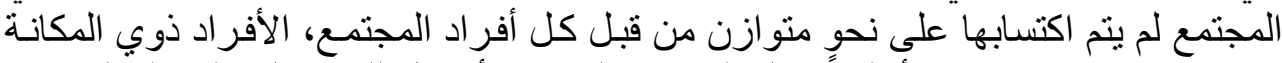

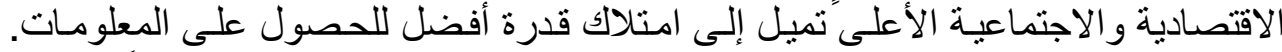
و هذا يقود إلى تقسيم المجتمع إلى صنفين هما مجموعة الناس والأفراد الأكثر تعليماً، و الذين الذين

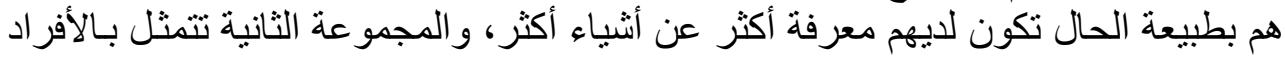

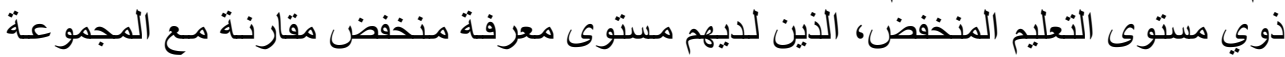

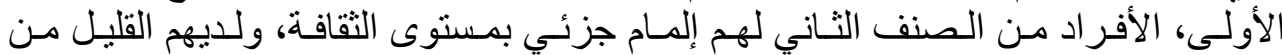

المعرفة بما يتعلق بالثؤون العامة (Holbrook, 2002, 437). 


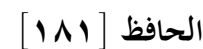

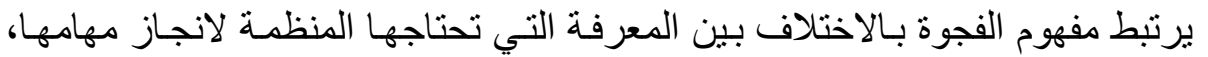

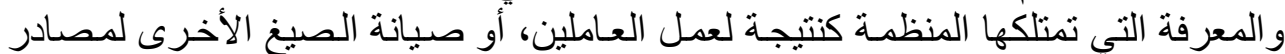

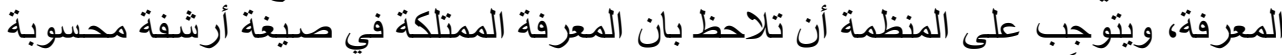

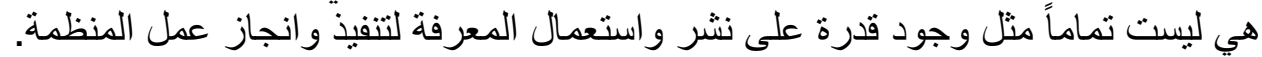

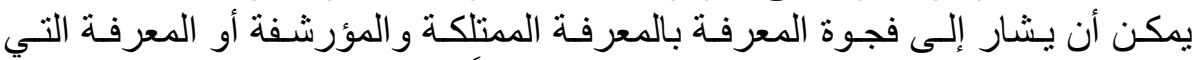

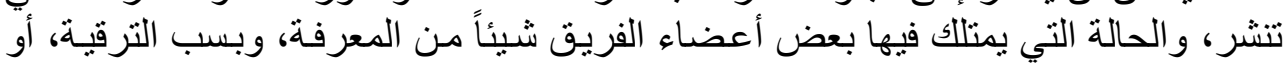

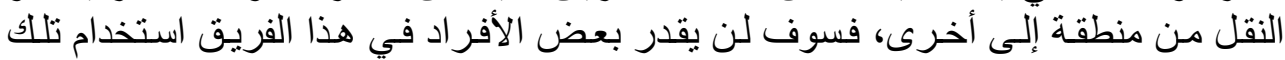

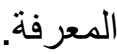

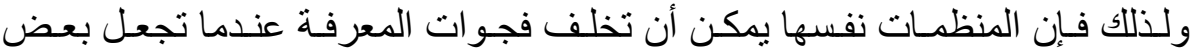

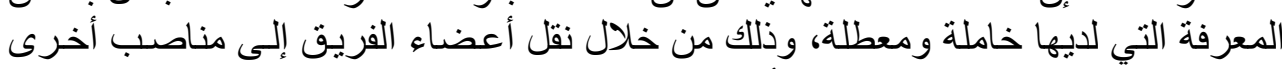

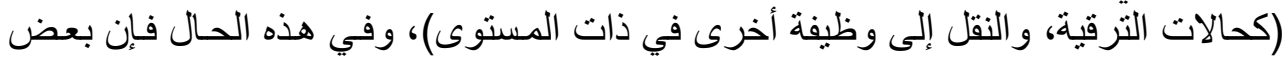

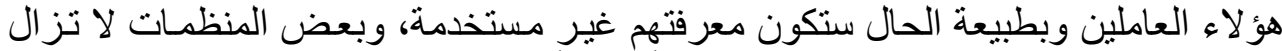

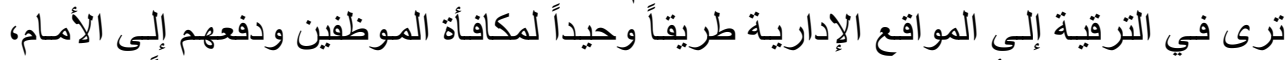

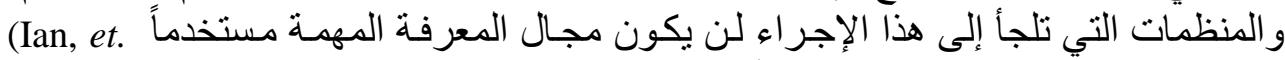

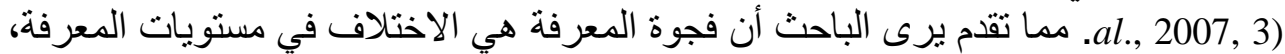

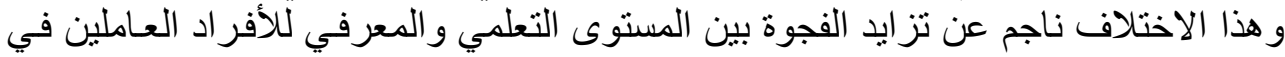

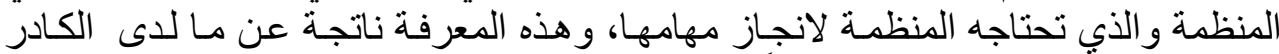

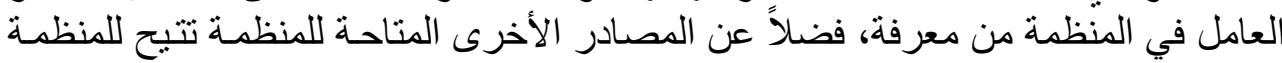
الحصول على المعرفة.

خامساً- أنواع فجوات المعرفة (وجهة نظر إستراتيجية)

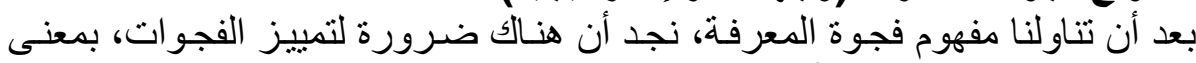

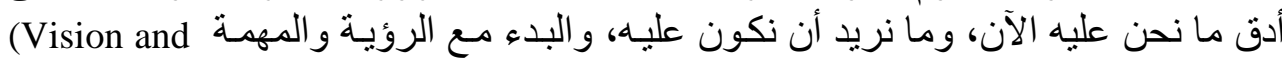
Mission)

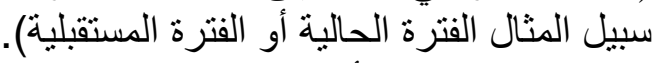

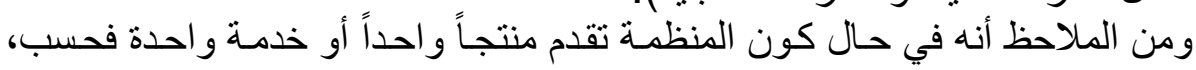
فإن الفجوة ستتكون من نوع واحد فقط، لكن إذا ما بحثت المنظمة في فتر الفرات مستقبلية، كما

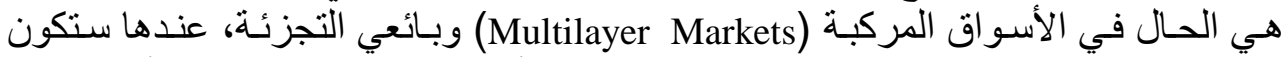

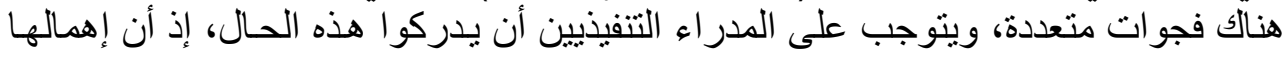
سيؤثر على مهام المنظمة (Russ, 2010, 14).

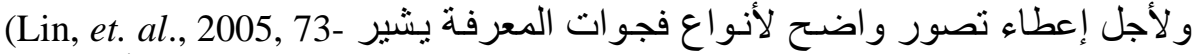

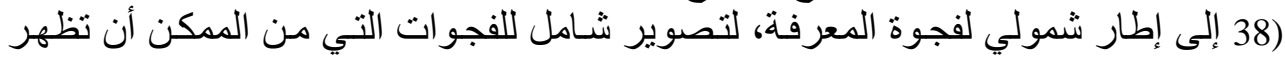

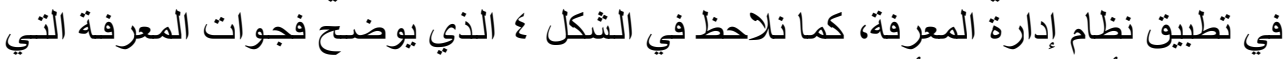
من الممكن أن نلاحظ في أربع سمات المات هي:

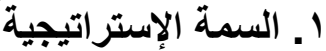

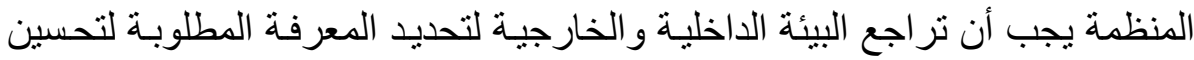

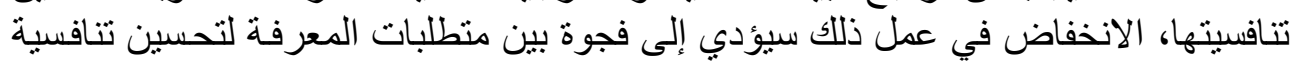

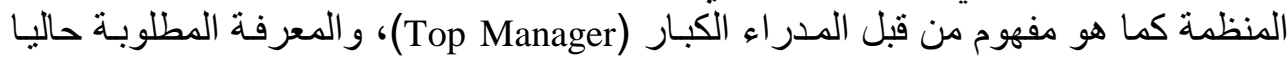




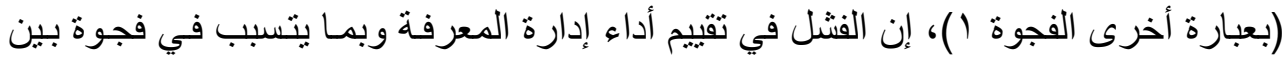

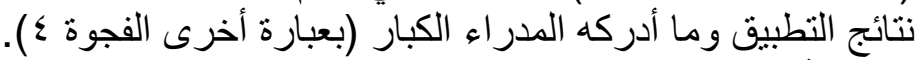

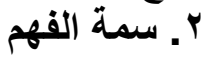

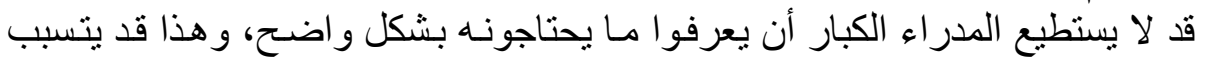

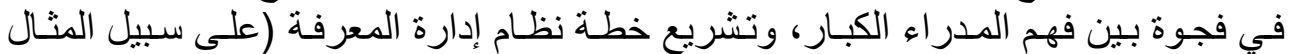

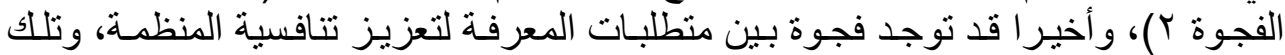

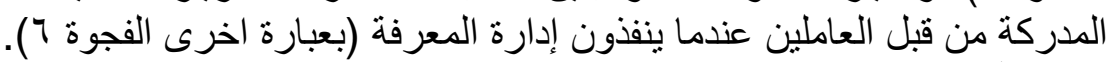

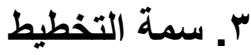

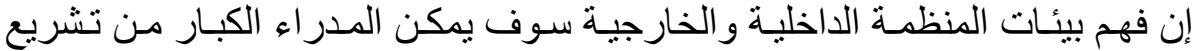
خطة صحية لتنفيذ إدارة المعرفة، وإذا كان المدر اء الكبار لا يستطيعون نقل هذه المعرفة المئة

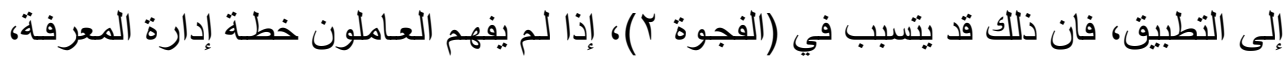

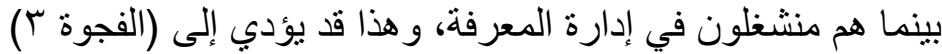

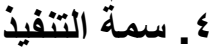

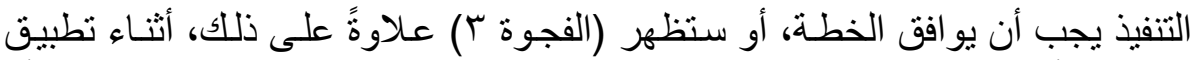

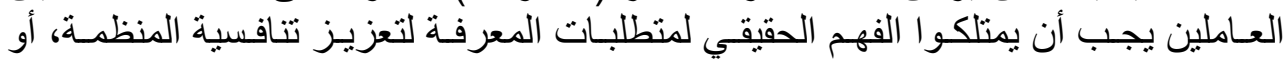
وعليه فإن تللك الفجوات الست الفئ تكون كما يأتي: ستظهر (الفجوة ع ).

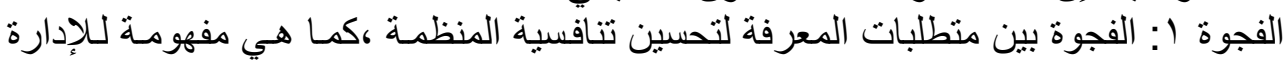
العليا واحتياجات المعرفة الفعلية لتعزيز منافينيها.

الفجوة r: الفجوة بين متطلبات المعرفة لتحسين تنافسية المنظمـة، كمـا هي مفهومـة للـإِدارة

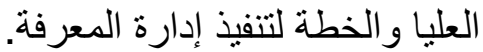

الفجوة ب: الفجوة بين خطة تتفيذ إدارة المعرفة كما هو مقترة الفرة من الإدارة العليا وتقدم التنفيذ لخطة إدارة المعرفة.

الفجـوة ع: الفجوة بـين المعرفـة التـي يـتم الحصول عليهـا بعـد تنفيـذ نظـام إدارة المعرفـة ومتطلبات المعرفة لتعزيز تتافسية المنظمة.

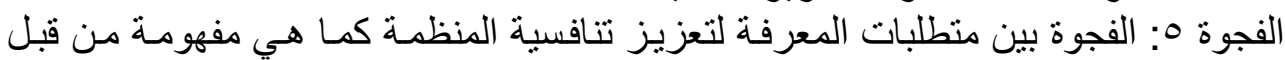

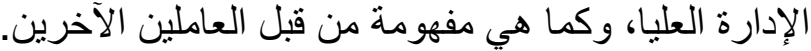

الفجوة 7: الفجوة بين منطلبات المعرفة لتعزيز تنافسية المنظمة كما تم فهمها.

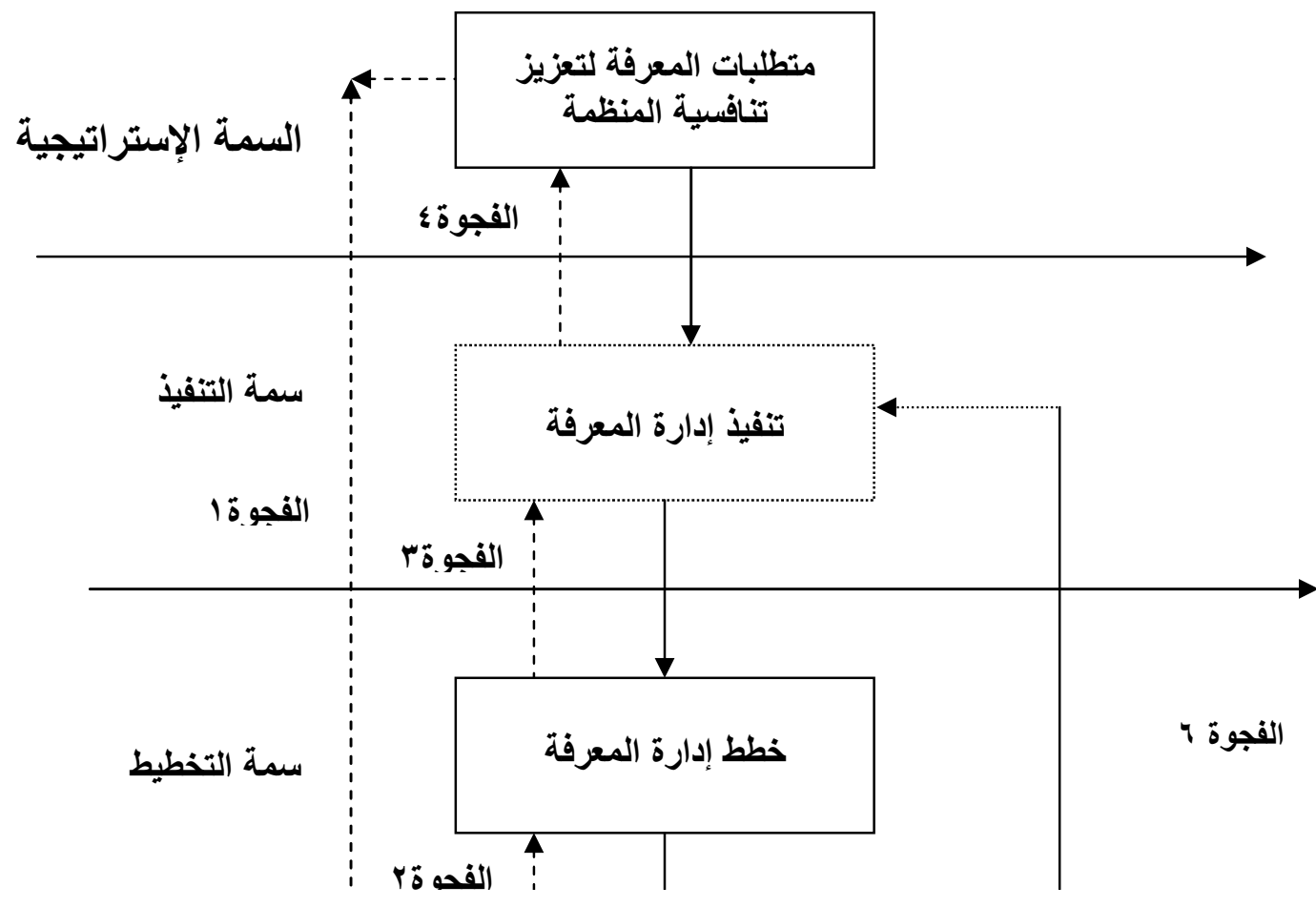


الحافظ [rir]

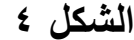 \\ أنموذج فجوات المعرفة}

Source: Cin, Chino. Yeh, Jong-Mau. Tseng. Shu-Mei, (2005), Case Study on Knowledge Management Gaps, Journal of Knowledge Strategy, Vol. 2, No. 3, (http:// crrm.4-3mrs. Fr/ blog/IMG)

سادساً تقليل الفجوة المعرفية (روية إستراتيجية)

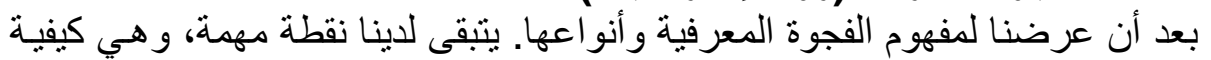

تقليل الفجوة المعرفيـة، وهنـا يشير (Russ et. al., 2010, 64) إلى مفهوم (KARMA) (Knowledge Assessment Review And Management Audit)

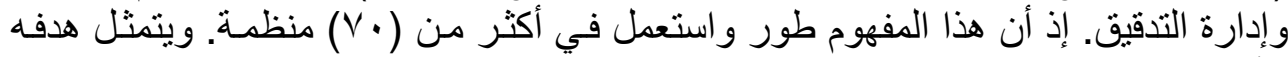

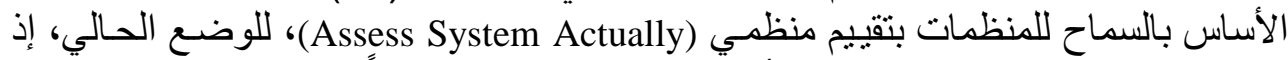

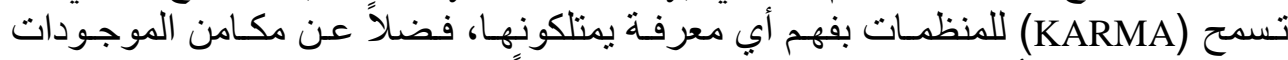

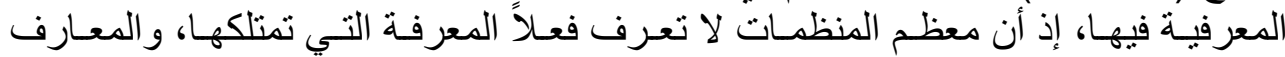

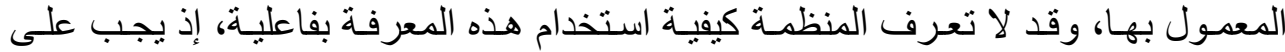

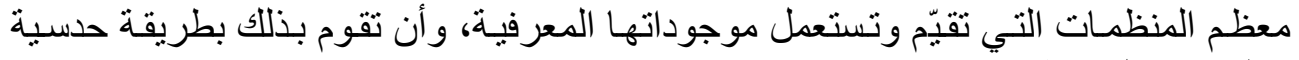

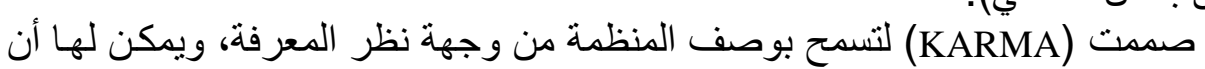

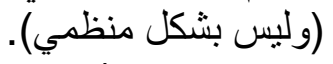

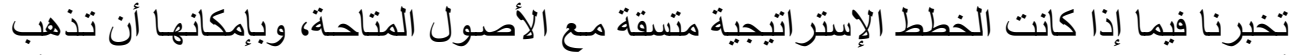

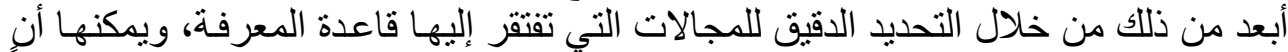

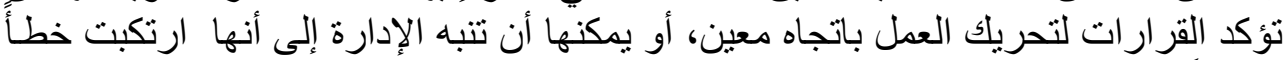


تتيح (KARMA) أساسـاً لتعقب مصدادر المعرفـة الداخلية والخارجيـة مـع الاهتمـام

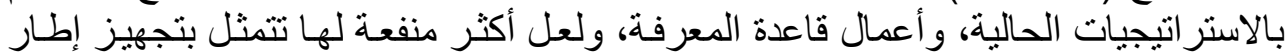

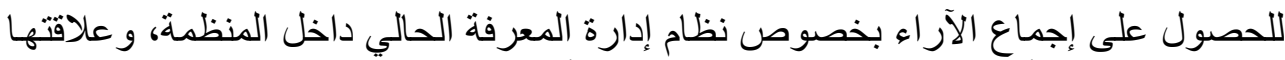

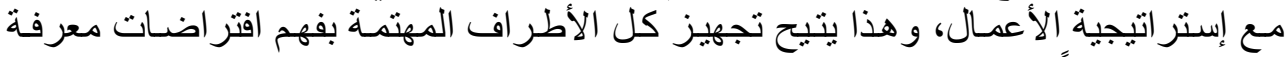

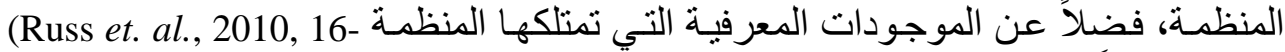

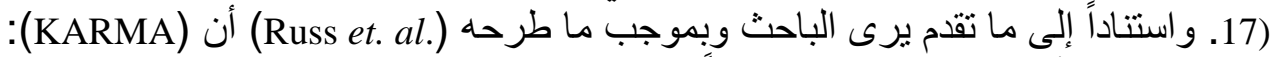

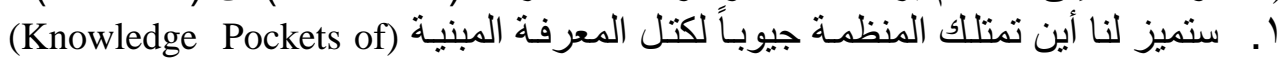
. Building Blocks)

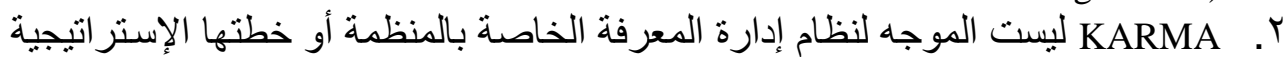

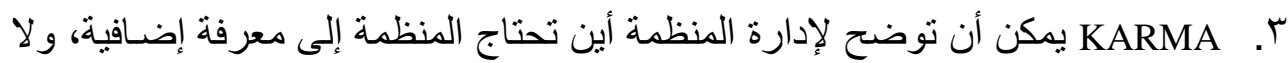
تسنطيع إخبار ها عن كيفية الحصول على تلادلك المعارف.

\section{الإطار الميداني}

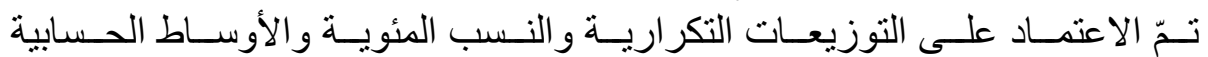

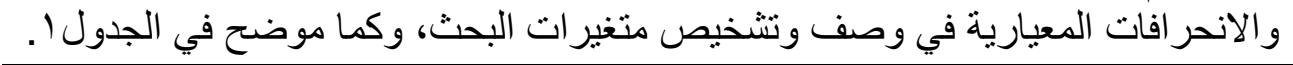

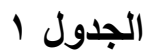

التوزيعات التكرارية والنسب المئوية والأوساط الحسابية والانحرافات المعيارية

لمتفيرات البحث

\begin{tabular}{|c|c|c|c|c|c|c|c|c|c|c|c|c|c|}
\hline \multirow{3}{*}{ المعياري } & \multirow{3}{*}{ الحسابي } & \multicolumn{10}{|c|}{ مقياس الاستجابة } & \multirow{3}{*}{ الرمز } & \multirow{3}{*}{$\begin{array}{l}\overline{3} \\
\overline{3}\end{array}$} \\
\hline & & \multicolumn{2}{|c|}{ لا أتثق بثدة } & \multicolumn{2}{|c|}{$\begin{array}{l}\text { لا أتثق) } \\
\text { (r) }\end{array}$} & \multicolumn{2}{|c|}{$\begin{array}{c}\text { محايد } \\
\text { (r) }\end{array}$} & \multicolumn{2}{|c|}{$\begin{array}{l}\text { أتفق) } \\
\text { (घ) }\end{array}$} & \multicolumn{2}{|c|}{$\begin{array}{c}\text { أتفق بثدة) } \\
\text { (0) }\end{array}$} & & \\
\hline & & $\%$ & $\Xi$ & $\%$ & 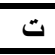 & $\%$ & $\Xi$ & $\%$ & $ت$ & $\%$ & $ت$ & & \\
\hline.$V Y 7$ & $r .071$ & - & - & IY.Y & 。 & $r \varepsilon \_$ & 1. & 01.0 & $r \varepsilon$ & ६.9 & $r$ & $X_{1}$ & \\
\hline$\Lambda \cdot \varepsilon$ & $\xi . \varepsilon \Omega \Lambda$ & & & $\overline{Y . r}$ & $\bar{r}$ & $\begin{array}{r}Y . Y \\
\end{array}$ & $r$ & 01.0 & $\overline{r \varepsilon}$ & $r \uparrow . \wedge$ & 11 & $\mathrm{X}_{2}$ & \\
\hline 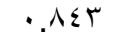 & $r \cdot \Lambda \cdot \varepsilon$ & - & - & ¿.A & r & r. & 14 & $\xi 1.0$ & IV & YY & 9 & $x_{3}$ & \\
\hline 174 & $\varepsilon . r 70$ & & & $\xi . \Lambda$ & $\bar{Y}$ & $r . \varepsilon$ & 1 & $\varepsilon \% .9$ & 11 & $\overline{r \wedge . \wedge}$ & $r$. & $\mathrm{X}_{4}$ & \\
\hline 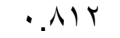 & E.IYT & - & & Y.r & $r$ & ६.१ & r & 04.1 & $r$ & $r I . V$ & 14 & $x_{5}$ & \\
\hline 1.10 & $r . r \varepsilon)$ & 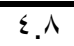 & r & $\overline{T r}$ & o & $r 9$ & 17 & $r \overline{r . V}$ & $\pi$ & $\overline{T r . r}$ & o & $\mathrm{X}_{6}$ & \\
\hline $1.1 \varepsilon \varepsilon$ & $r .190$ & $\overline{\nu . \Gamma}$ & $r$ & $r Y$ & 9 & $\overline{r \uparrow . \Lambda}$ & 11 & $\overline{r 1 .}$ & $\pi$ & $\overline{T Y . r}$ & 0 & $\overline{X_{7}}$ & $\frac{3}{5}$ \\
\hline$\cdot .170$ & r.६ईई & - & - & $\frac{19.1}{1 Y .1}$ & $\bar{v}$ & $r \bar{r}{ }^{\prime}$ & $\overline{T r}$ & $\varepsilon r .9$ & 11 & $\frac{V . r}{r}$ & $r$ & $\mathrm{X}_{8}$ & \\
\hline$\cdot . V Y 7$ & 5.071 & - & - & $\bar{r}, r$ & $r$ & rq & 17 & $\varepsilon r . q$ & 11 & 9.1 & $\xi$ & $\mathrm{X}_{9}$ & \\
\hline .911 & $r .7 .9$ & 9.1 & $\bar{\varepsilon}$ & $r 9$ & 17 & $\overline{r ! . \nu}$ & $\pi$ & 19.0 & $\Lambda$ & - & - & $\mathrm{X}_{10}$ & \\
\hline $1 . . \Delta Y$ & r.TA & IV.1 & v & $r\{, \xi$ & 1. & $r \varepsilon .1$ & $1 \varepsilon$ & YY & 9 & r. & 1 & $X_{11}$ & \\
\hline.$Y Y \xi$ & $\varepsilon . r \varepsilon$ & - & - & T. $\varepsilon$ & $T$ & $\overline{I V . V}$ & v & 04.1 & $\overline{Y r}$ & 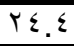 & 1. & $\mathrm{X}_{12}$ & \\
\hline . & $8,1 T Y$ & 9.1 & $\xi$ & $1 Y .1$ & $\bar{v}$ & $r q, r$ & $T$ & $\overline{r I V}$ & $\frac{\pi}{14}$ & $\frac{\gamma r}{\gamma}$ & $r$ & $\mathrm{X}_{13}$ & \\
\hline .099 & $r . \wedge \vee \lambda$ & - & & T. . & 1 & $I Y . Y$ & V & $V \cdot . v$ & rq & 9.1 & $\varepsilon$ & $\mathrm{X}_{14}$ & \\
\hline..$\Lambda \uparrow \Lambda$ & T.TYT & & & & & & & $O V_{. V}$ & & & & الكلي & المع الم \\
\hline 1.09 & r.T & $\bar{\gamma}, \bar{r}$ & $r$ & IT.Y & 0 & $r \mid . v$ & $\pi$ & rq & 17 & 9.1 & $\xi$ & $X_{15}$ & - \\
\hline
\end{tabular}


الحافظ [110]

\begin{tabular}{|c|c|c|c|c|c|c|c|c|c|c|c|c|c|}
\hline \multirow{3}{*}{ الالحعراف } & \multirow{3}{*}{ الحسابي } & \multicolumn{10}{|c|}{ مقياس الاستجابة } & \multirow{3}{*}{ الرمز } & \\
\hline & & \multicolumn{2}{|c|}{ لا أتفق بثدة } & \multicolumn{2}{|c|}{$\begin{array}{l}\text { لا أتفق } \\
\text { (r) }\end{array}$} & \multicolumn{2}{|c|}{$\begin{array}{l}\text { محايد } \\
\text { (r) }\end{array}$} & \multicolumn{2}{|c|}{$\begin{array}{l}\text { أتفق } \\
\text { (؛) }\end{array}$} & \multicolumn{2}{|c|}{$\begin{array}{c}\text { أتفق بشدة } \\
\text { (0) }\end{array}$} & & 柔 \\
\hline & & $\%$ & $\Xi$ & $\%$ & $ت$ & $\%$ & $ت$ & $\%$ & $\Xi$ & $\%$ & ت & & \\
\hline$\cdot .719$ & 5.519 & - & - & Y. & 1 & $\overline{V . r}$ & $r$ & 07.1 & $r \mu$ & $r \leqslant .1$ & $1 \varepsilon$ & $X_{16}$ & \\
\hline $1 . T V$ & $r .01 Y$ & $\overline{r . \varepsilon}$ & $T$ & $1 \varepsilon .7$ & 7 & $r q . r$ & $T$ & r. & 10 & IV.1 & $\bar{v}$ & $X_{17}$ & \\
\hline .9 .1 & E.Y YY & T. $\varepsilon$ & $T$ & T. $\varepsilon$ & $T$ & $\overline{V . r}$ & $r$ & $r 9$ & 17 & $\varepsilon \Lambda . \wedge$ & $r$. & $\mathrm{X}_{18}$ & \\
\hline$\cdot$. Ат. & $\varepsilon .97$ & $\overline{r . \varepsilon}$ & $T$ & $\overline{r . \varepsilon}$ & $T$ & 9.1 & $\varepsilon$ & or.V & $\overline{Y Y}$ & YI.V & 14 & $\mathrm{X}_{19}$ & \\
\hline$\cdot . \mathrm{NVT}$ & $\sum .190$ & T.乏 & 1 & - & - & $1 \leq .7$ & 7 & $\sum 1.0$ & IV & $\leqslant 1.0$ & IV & $\mathrm{X}_{20}$ & \\
\hline$\cdot . \Lambda \Lambda Y$ & r.10T & T.乏 & 1 & T4.7 & 10 & M. & 10 & YY & 9 & T.乏 & 1 & $\mathrm{X}_{21}$ & \\
\hline$\cdot . \Sigma 71$ & $\varepsilon . v T$ & - & - & - & - & V.r & $r$ & $V \lambda$ & rY & $1 \leq .7$ & 7 & $\mathrm{X}_{22}$ & \\
\hline $1 . \cdot \varepsilon r$ & r.V07 & $\overline{r . \varepsilon}$ & $T$ & 9.1 & $\varepsilon$ & 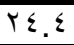 & 1. & r. & 10 & 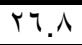 & 11 & $\mathrm{X}_{23}$ & \\
\hline$\because A V Y$ & 5.190 & - & - & $\overline{Y . Y}$ & $\bar{r}$ & $\overline{Y . r}$ & $r$ & $\varepsilon r .9$ & 11 & $\sum 1.0$ & IV & $\mathrm{X}_{24}$ & \\
\hline$\cdot . \wedge \wedge \theta$ & r.A01 & & & & & & & $\overline{v 1.0}$ & & & & الكلي & المعد المدا \\
\hline
\end{tabular}

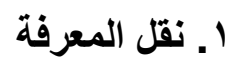

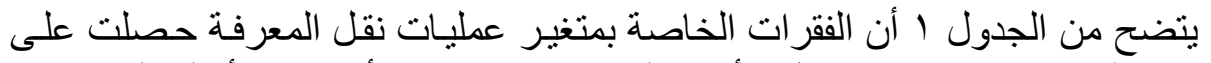

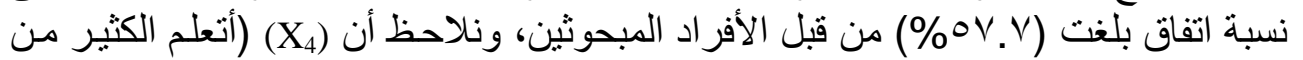

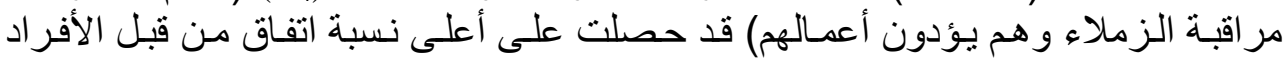

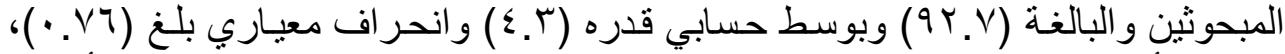

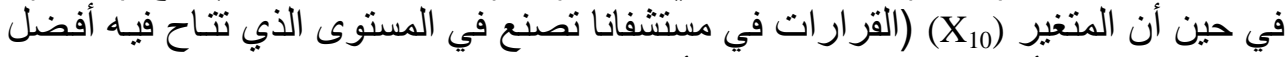

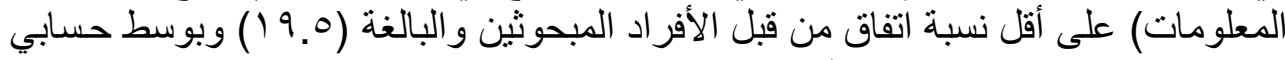

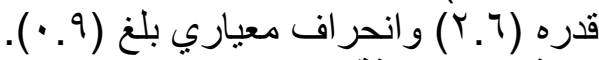

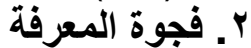

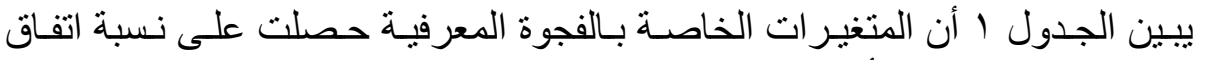

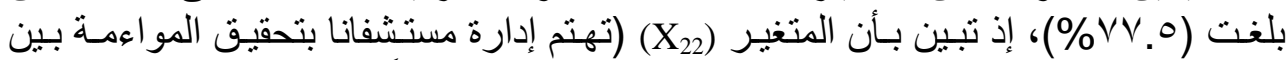

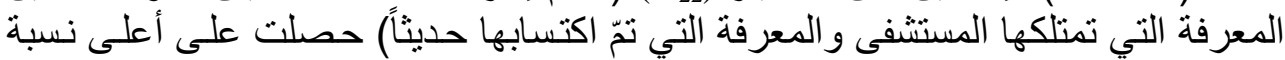

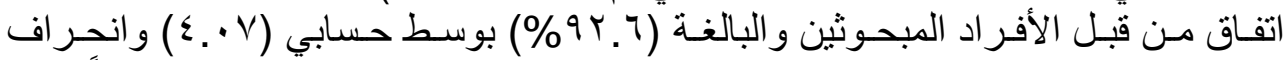

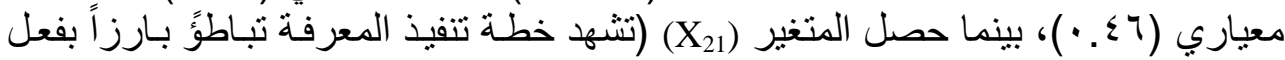

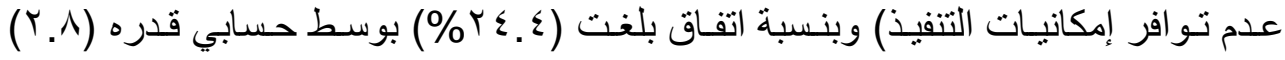

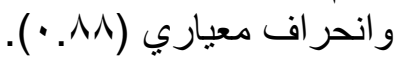

ثانياً - علاقات الارتباط بين متغيرات البحث

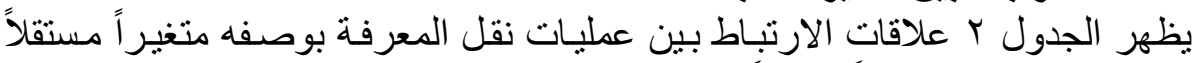
وفجوة المعرفة بوصفها متغير اً معتمداً.

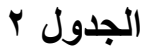

علاقات الارتباط بين عمليات نقل المعرفة وفجوة المعرفة

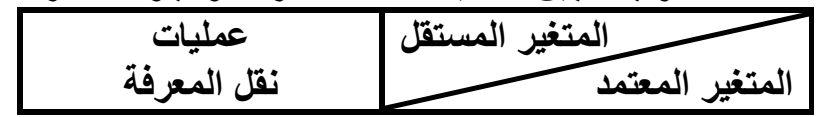




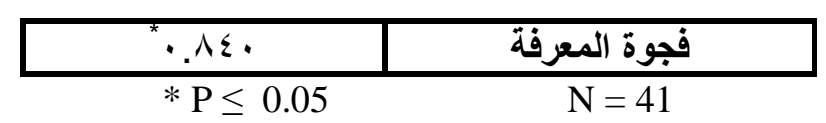

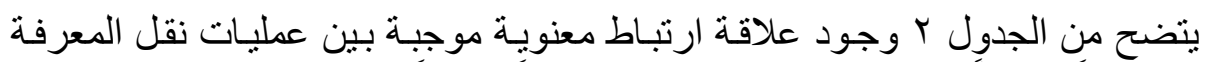

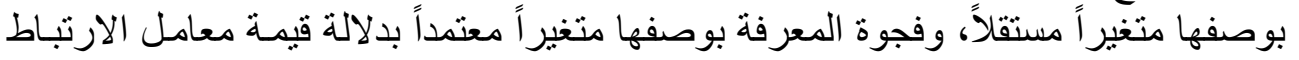

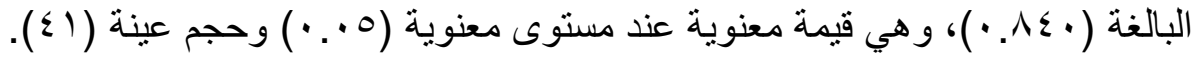

ثالثاً.علاقات التأثير بين متغيرات الاراسة

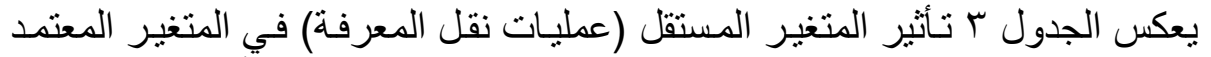

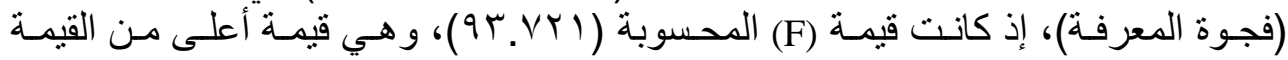

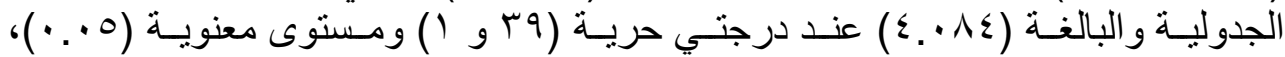

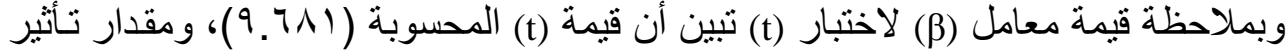

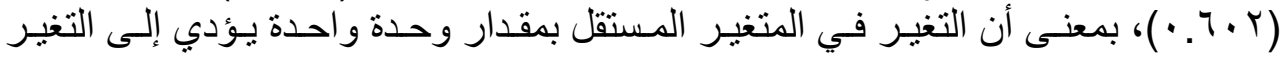

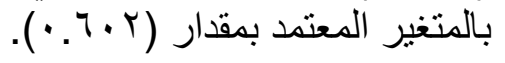

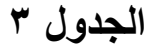

علاقات التأثثير بين متغيرات الارل استة

\begin{tabular}{|c|c|c|c|c|c|}
\hline \multicolumn{2}{|c|}{$\mathbf{F}$} & \multirow{2}{*}{$\mathbf{R}^{2}$} & عمليات نقل المعرفة & \multirow{2}{*}{$\beta_{0}$} & \multirow{2}{*}{ المتغير المعتئما المستقل } \\
\hline جلولية & محسوية & & $\beta_{1}$ & & \\
\hline$\varepsilon . \wedge \varepsilon$ & $9 T . V Y)$ & $\cdot V \cdot 7$ & 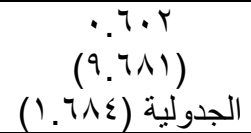 & $1 . \varepsilon \vee \wedge$ & فجوة المعرفة \\
\hline
\end{tabular}

رابعاً- فجوات المعرفة بحسب مستوياتها في المستثفيات المبحوثة

تثنير الفقرات (X)

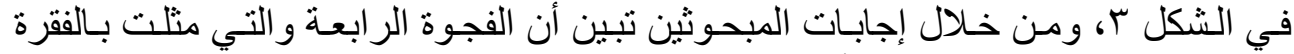

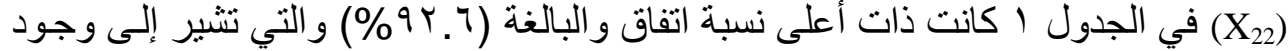

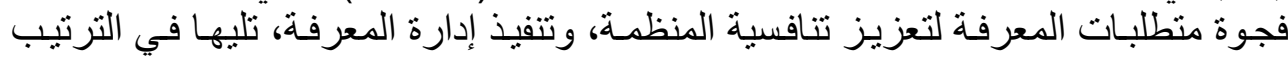

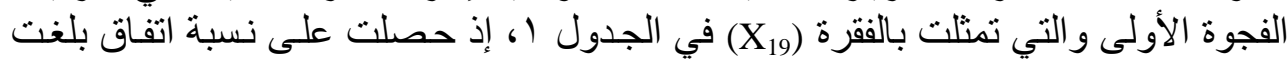

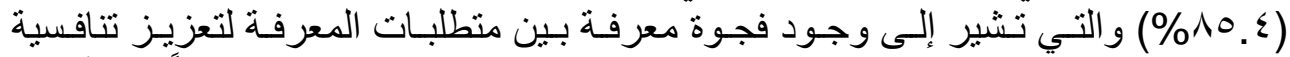

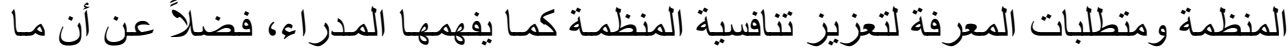

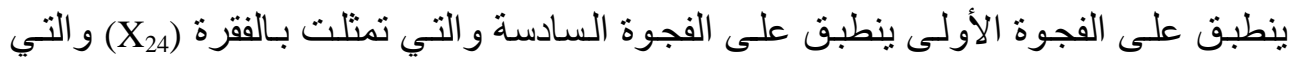

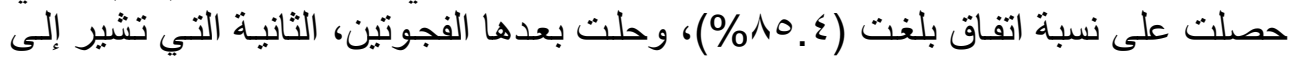

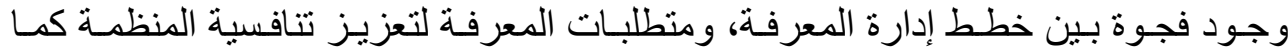

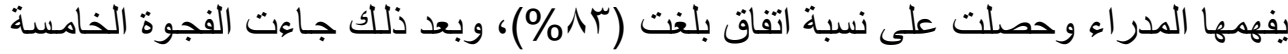

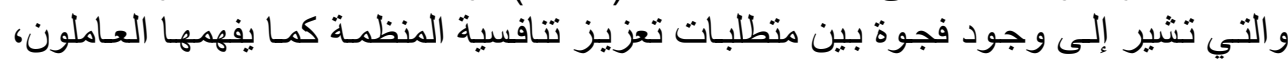

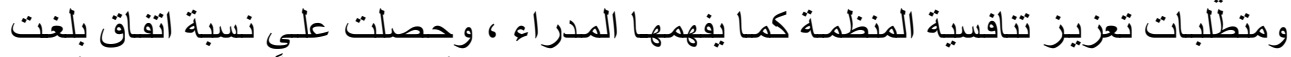

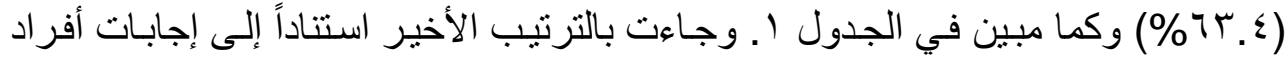




\section{[1Av] الحافظ}

عبنـة البحث، الفجوة الثالثـة تشير إلى وجود فجوة بين تتفيذ إدارة المعرفـة وخطط إدارة

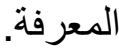

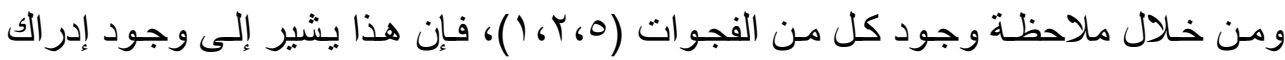

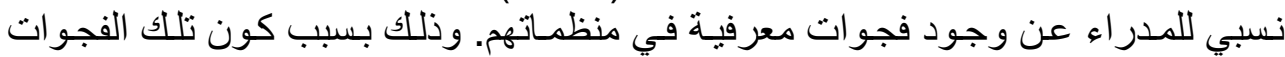
متعلقة أساسا بتصورات المدر اء عن المعرفة التي المتاحة في منظمـاتهم، إذ تشير الفجوة

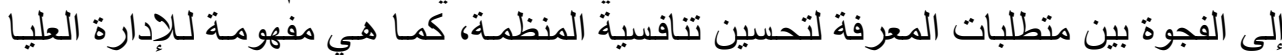

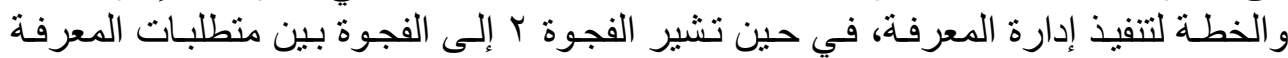

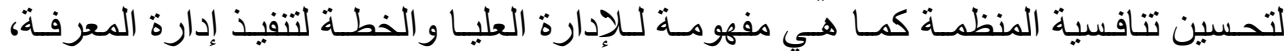

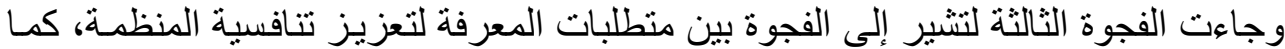
هي مفهومة من قبل الإدارة العليا، وكما هي مفهومة من قبل العاملين الآخرين

الاستنتاجات و المقترحات

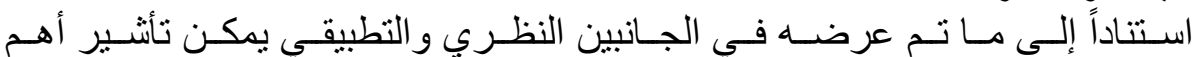
الاستتناجات و التوصيات، وكما يأتي:

أولاًا-الاستتنتاجات

ا. . تعدّ عمليات نقل المعرفة من المداخل الحديثة التي تسعى إلى تحقيق نقل نـاجح للمعرفة

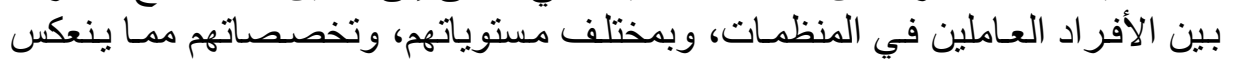
بشكلٍ مباشر على فجوة المعرفة باتجاه تقليلها إلى أدنى مستوى للى للاستفادة من المعرفة

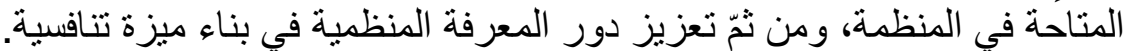

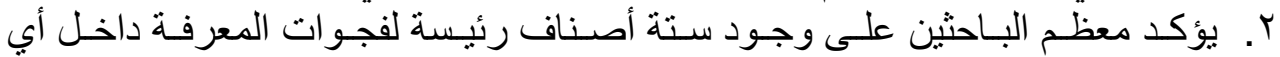

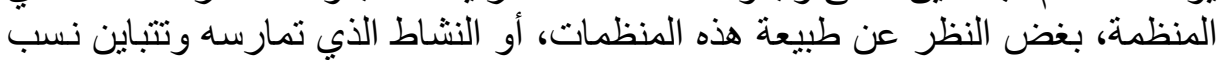

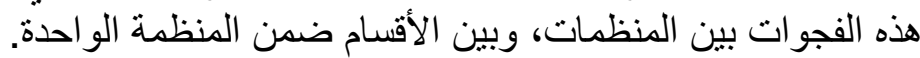

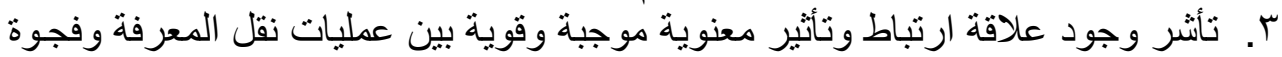

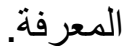

ع. ثبت أن عمليات نقل المعرفة تؤدي إلى تقليل فجوات المعرفة في المستشفيات عينة الدر اسة بدليل قيم علاقات الارتباط و التأثير التي ظهرت في في في في التحليل.

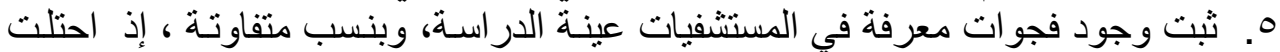

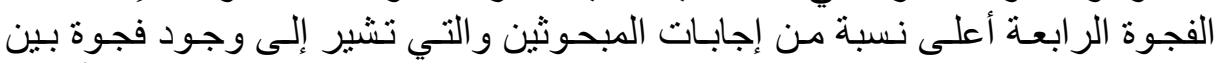

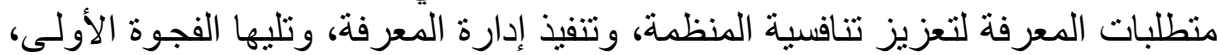

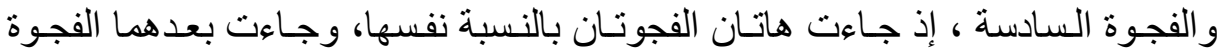

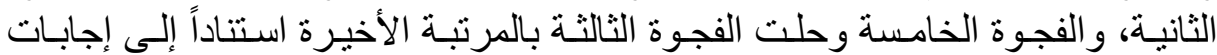

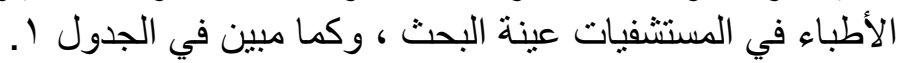

بناء على الاستنتاجات التي تمّ التوصل إليها آنفاً، يمكن عرض المناء المقترحات الآتية:

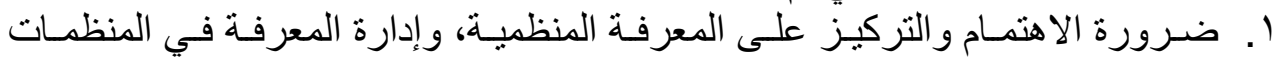

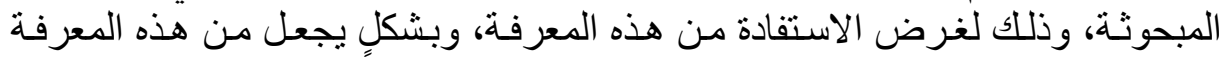
ميزة تنافسية، تمكن المنظمات من التنافس مع مثيلاتها في المنظمات الأخرى الخرى 


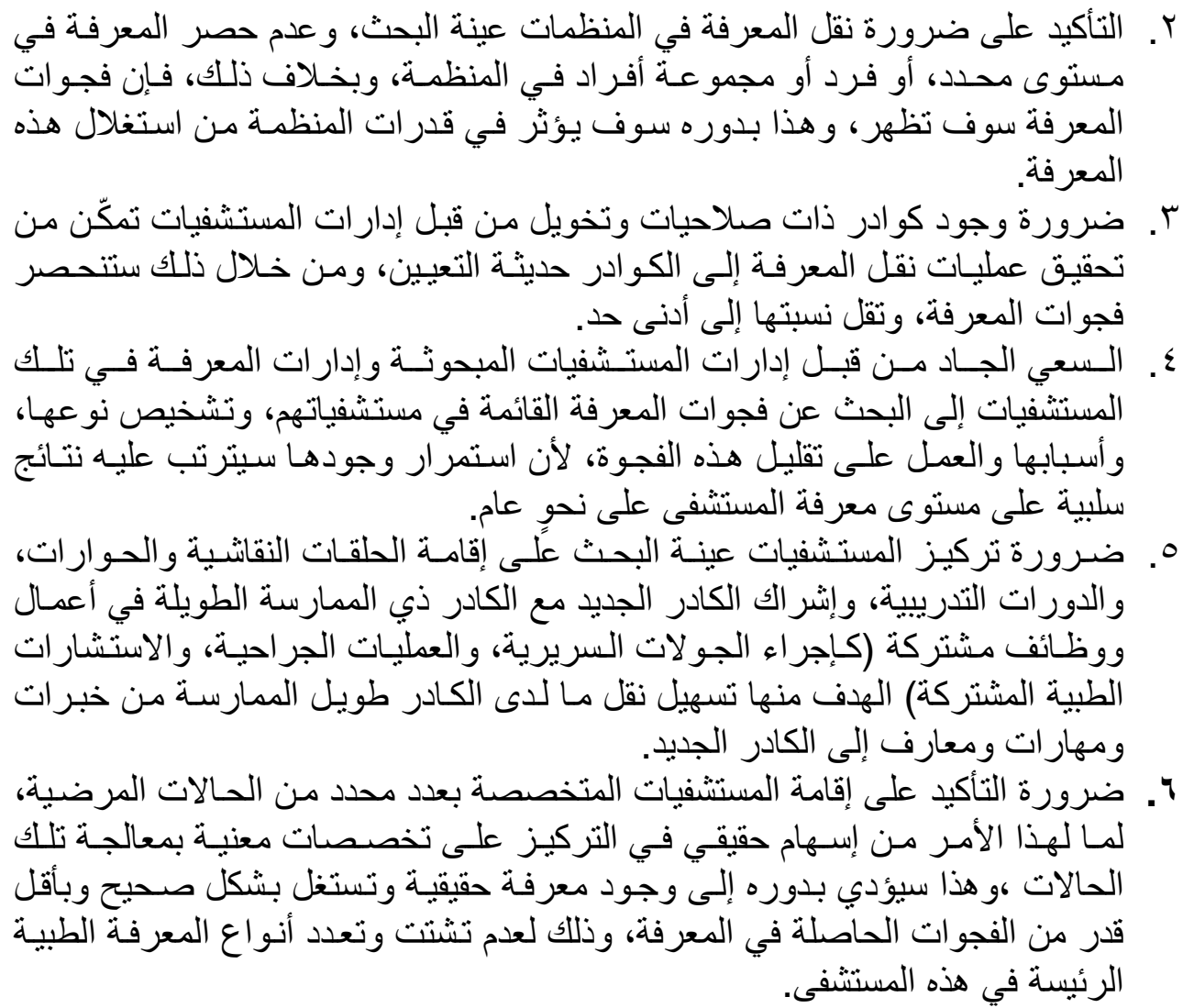

\section{Reference}

1. Argot, I. and Ingram P., 2000 Knowledge Transfer: A Basis for Competitive Advantage in Firms. Organization Behavior and Human Decision Processes, 821.

2. Bratianu, Constantin, Vas. Lache, Simona, Stancor, Vihalie, 2009, Knowledge Transfer Processes in Romanian Multinational Companies, Review of International Comparative Management, Vol. 10. Issue 1, www.rmci:ase.org

3. Brochner, J. R. Osander and Waara F., 2004, Cross-Border Post-Acquisition Knowledge Transfer Among Construction Management and Economic, 22.

4. Cin, Chinho, Yeh. Jong-Mau.Tseng, Shu-Mei, 2005, Case Study on Knowledge Management Strategy, Vol. 1, No. 3. http://crrm.u-3mrs.fr/blog/IMG

5. Cummings J. I and Teng, B., 2003, Transferring RandD Knowledge: The Key Factor Affecting Knowledge Transfer Success, Journal of Engineering and Technology Management.

6. Dougherty, 1999, Industrial and Commercial Training, No. 1019.7858, Vol. 3, No. 7. MC B University Press.

7. Elinfoo. K. Harriet 2005, Knowledge Creation and Knowledge Transfer in Construction Organization in Tanzania. Doctoral Thesis, Royal Stitue of Technology. Stokholm, Sweden. www.kth.se. polopoly-f.com

8. Eraut, Michael 2007, Theoretical and Practical Knowledge Revisited, University of Sussex. www.mofet.macam.ac.il

9. Harlicek, Ticha, 2007, Knowledge Transfer Agric, Econ.czecH.53 $2007 \quad 12$ www.Journalz. Uzpi.cz 


\section{[1/19] الحافظ]}

10. Hirsh, Wendy, Eraut, Michael, 2007, The Significance of Work Place Learning for Individuals. Groups and Organization. www.skope.ox.al.uk.

11. Hol Brook. T.M, 2002, President Campaigns and the Knowledge Gap. Political Communities. 19. Online. Or the World Wide. www.polisici.taylorand taylorandlfrancis.com

12. http//: bluewirecst.tzo.com:2004

13. Leven, David I.and Gilbert. April, 1998, Knowledge Transfer Management Practices Underlying One Piece of the Learning Organization http//:faculty.hass.berkely.edu

14. McBriar. Smith Colin, Bain Geoff. Un Swortn, Peter. Lancashire, Blackburn. Magraw Stephen. Gordon John. 1., 2007, Risk, Gap and Strength: Key Concepts in Knowledge Management, www.akri.org.

15. Ruck, 2006, Independent External Challenge Report To Research Council UK, Knowledge Transfer in the Eight Research Councils, London, Research Councils. UK.

16. Russ, Mire, 2010, Knowledge Management Strategies for Business Development, Business Science Reference, Hershey. New York IGI. Global. USA. www.igiglobal.com.

17. Szulanski, G. 2003, Sticky Knowledge: Barriers of Knowledge in the Firm, London. SAGE Publications.

18. Vote, A. Garavello, A. C. and Schiuma, G., 1999, Knowledge Transfer and Inter-firm Relationship in Industrial Districts: The Role of Leader Firm, Techno Ration, Vol. 19.

19. Wilkes Mann, U. We, Virgillito, Alfredo, Wilkes Mann, Maxi Miliane, 2009, Knowledge Management As Second Level Management, www.zfw.uni-de.

20. Wilkes Mann, U. We, Wilkes Mann, Maxi Milian, Virgillito, Alfredo, 2007, Requirements for Knowledge Transfer in Hospital / How Can Knowledge Transfer be Supported in Hospitals? www.zfw.uni.dortmund.

21. www.leading.com 2009

22. www.regions 4resarch.eu. 